NBER WORKING PAPER SERIES

\title{
SMALL AND LARGE PRICE CHANGES AND THE PROPAGATION OF MONETARY SHOCKS
}

\author{
Fernando Alvarez \\ Hervé Le Bihan \\ Francesco Lippi \\ Working Paper 20155 \\ http://www.nber.org/papers/w20155
NATIONAL BUREAU OF ECONOMIC RESEARCH
1050 Massachusetts Avenue
Cambridge, MA 02138
May 2014

We benefited from the comments of Manuel Amador, Saroj Bhattarai, Ricardo Caballero, V.V. Chari, Mike Dotsey, Jordi Galí, Aubik Kahn, Ricardo Caballero, Virgiliu Midrigan, Daniel Levy, Nicola Pavoni, Giorgio Primiceri, Jon Steinsson, Julia Thomas, Michael Waterson, Ivan Werning, and seminar participants at the 2014 NBER EF\&G meeting in NY, Chicago Fed, Minneapolis Fed, and the following universities: Bocconi, Bologna, Firenze, MIT, Ohio State, HECER and Lausanne. We are grateful to Alberto Cavallo, Pete Klenow, Oleksiy Kryvtsov, and Joseph Vavra for providing us with several statistics not available in their papers. We thank David Argente and Fredrik Wulfsberg for proving us with evidence for the US scanner data and Norway's CPI (respectively). We thank the INSEE and the SymphonyIRI Group, Inc. for making the data available. All estimates and analysis in this paper, based on data provided by SymphonyIRI Group, Inc. are by the authors and not by SymphonyIRI Group, Inc. We thank the Fondation Banque de France for supporting this project. Part of the research for this paper was sponsored by the ERC advanced grant 324008. The views expressed in the paper are of the authors and do not necessarily reflect those of the Banque de France. Roberto Robatto provided excellent research assistance. The views expressed herein are those of the authors and do not necessarily reflect the views of the National Bureau of Economic Research.

At least one co-author has disclosed a financial relationship of potential relevance for this research. Further information is available online at http://www.nber.org/papers/w20155.ack

NBER working papers are circulated for discussion and comment purposes. They have not been peerreviewed or been subject to the review by the NBER Board of Directors that accompanies official NBER publications.

(C) 2014 by Fernando Alvarez, Hervé Le Bihan, and Francesco Lippi. All rights reserved. Short sections of text, not to exceed two paragraphs, may be quoted without explicit permission provided that full credit, including $\odot$ notice, is given to the source. 
Small and Large Price Changes and the Propagation of Monetary Shocks

Fernando Alvarez, Hervé Le Bihan, and Francesco Lippi

NBER Working Paper No. 20155

May 2014

JEL No. E3

\section{ABSTRACT}

We document the presence of both small and large price changes in individual price records from the CPI in France and the US. After correcting for measurement error and cross-section heterogeneity, the size-distribution of price changes has a positive excess kurtosis. We propose an analytical menu cost model that encompasses several classic models, as Taylor (1980), Calvo (1983), Reis (2006), Golosov and Lucas (2007) and accounts for observed cross-sectional patterns. We show that the ratio of kurtosis to the frequency of price changes is a sufficient statistics for the real effects of monetary policy in a large class of models.

Fernando Alvarez

University of Chicago

Department of Economics

1126 East 59th Street

Chicago, IL 60637

and NBER

f-alvarez1@uchicago.edu

Hervé Le Bihan

Banque de France

DCPM SEMAP

DGEI 41-1391

31 rue Croix des Petits Champs

75049 Paris Cedex 01

France

herve.lebihan@banque-france.fr
Francesco Lippi

EIEF

Via Sallustiana 62

Rome, Italy

flippi@uniss.it

An online appendix is available at:

http://www.nber.org/data-appendix/w20155 


\section{Introduction}

This paper provides an analytical characterization of the steady state equilibrium and of the response of output to an unexpected monetary shock for a class of models with sticky prices. By combining the assumptions of multiproduct firms and random menu costs the model is able to produce, in various degrees, both the small and large price changes that have been documented in the micro data starting with Kashyap (1995). Different sticky price set-ups, spanning the models of Taylor (1980), Calvo (1983), Golosov and Lucas (2007), some versions of the "CalvoPlus" model by Nakamura and Steinsson (2010), the "rational inattentiveness" model by Reis (2006), as well as the multi-product models of Midrigan (2011), Bhattarai and Schoenle (2014) and Alvarez and Lippi (2014), are nested by our model. This unified framework allows us to unveil which assumptions are required to obtain each of them as an optimal mechanism.

The main analytical result of the paper is that, in a large class of models that extends those listed above, the total cumulative output effect of a small unexpected monetary shock depends on the ratio between two steady-state statistics: the kurtosis of the size-distribution of price changes $\operatorname{Kur}\left(\Delta p_{i}\right)$ and the average number of price changes per year $N\left(\Delta p_{i}\right)$. Formally, given the labor supply elasticity $1 / \epsilon$ and a small monetary shock $\delta$, we show that the cumulative output $\mathcal{M}$, namely the area under the output impulse response function, is given by

$$
\mathcal{M}=\frac{\delta}{6 \epsilon} \frac{\operatorname{Kur}\left(\Delta p_{i}\right)}{N\left(\Delta p_{i}\right)}
$$

The impact of the frequency $N\left(\Delta p_{i}\right)$ on the real output effect is understood in the literature, albeit not in such a stark fashion as in equation (1), and motivates a large body of empirical literature. The main novelty is that the effect of $\operatorname{Kur}\left(\Delta p_{i}\right)$ is equally important, and motivates our interest to review and extend the evidence on its measurement. For a symmetric distribution kurtosis is a scale-free statistic describing its shape, specifically its peakedness: the extent to which "large" and "small" observations (in absolute value) appear relative to intermediate values. We show that this statistic embodies the extent to which "selection" of price changes occurs. The selection effect, a terminology introduced by Golosov and Lucas (2007), indicates that the size of the price changes after the shock is not taken at random in the cross section of firms: in their menu cost model the price changes immediately after a shock are also the largest, so that the CPI response is fast. Instead, in Calvo the size of the average price change following a shock is constant, so that the CPI rises more slowly and the real effects are six times larger. Surprisingly, these features are fully captured by kurtosis, as in equation (1), since it is the mass in the proximity of the adjustment barriers 
that determines the nature of the price changes following a monetary shock.

We show that a selection effect also operates relative to the timing, not just the size, of price changes. For instance in the models of Taylor and Calvo, calibrated to the same frequency $N\left(\Delta p_{i}\right)$, the size of the price changes is the same (so there is no selection in the Golosov-Lucas sense). Yet the real cumulative output effect in Calvo is twice the effect in Taylor. This happens because in Taylor the distribution of times until adjustment is uniform, but in Calvo it is exponential, with a thicker right tail of firms that adjust very late. This selection effect concerning the times of adjustment is also captured by equation (1), since a more dispersed distribution of times to adjust produces a distribution of price changes that is a mixture of normals with different variances, and hence a higher kurtosis.

The main advantage of equation (1) is robustness: the formula allows us to discuss the output effects of small monetary shocks without having to solve for the whole general equilibrium or providing details about several other modeling choices. We see this result in the spirit of the sufficient statistic approach introduced in the public finance literature by Chetty (2009) and applied to the new trade literature by Arkolakis, Costinot, and Rodriguez-Clare (2012): in a nutshell, the identification of a robust relationship that contains useful economic information independently of many details of the model and is, at least in principle, observable in the data. Of course equation (1) does not hold in all models. For instance, it does not hold in models where the steady state distribution of price changes is not informative about the cost and benefits of changing prices. The simplest example is a model with no menu costs in which (with zero inflation) the distribution of price changes mirrors the distribution of the underlying cost shocks. Yet, if there is a monetary shock, all prices change immediately, and there is no effect on output. ${ }^{1}$ In those models additional information is needed to identify the parameters and assess the effects of monetary shocks.

\section{Overview}

We begin the paper with a review of the micro evidence on price setting behavior, across several micro datasets, and then develop a theoretical model that is able to qualitatively replicate the observed patterns. The empirical contribution documents the presence of small and large price changes, i.e. a very peaked distribution of price changes, using a dataset of price records underlying the French CPI as well as several US datasets. This finding, reminiscent of Kashyap (1995) seminal investigation of selected catalog retail goods, persists even at a very disaggregate level of product-outlet-type, ruling out an explanation based on

\footnotetext{
${ }^{1}$ While this example is extreme, the same logic applies in a model with a small menu cost and infrequent shocks to production costs that are similar to those observed in empirical distribution of price changes (see Appendix $\mathrm{H}$ for details).
} 
pure cross-section heterogeneity, and it is similar to Klenow and Kryvtsov (2008) analysis of the US CPI data. We also acknowledge that the CPI data may contain measurement error that tends to distort the measure of peakedness of the distribution of price changes, an issue emphasized by Eichenbaum et al. (2014). We estimate that -after taking into consideration measurement error and cross-sectional heterogeneity- the shape of the size-distribution of price changes is in between a Normal and a Laplace distribution, with a kurtosis that is about 4 for the US and about 5 for France.

We develop an analytical model that matches these patterns, featuring both the small and large price changes which lead to excess kurtosis. The model extends the multi-product setup developed in Alvarez and Lippi (2014), where the fixed menu cost applies to a bundle of $n$ goods sold by each firm. Each good is subject to idiosyncratic cost shocks that create a motive for price adjustment. The shocks are uncorrelated across goods and we assume zero inflation, i.e. lack of a common drift. The multi-product assumption generates the extreme price changes, both small and large. We extend that setup by introducing random menu costs, a feature that produces a positive excess kurtosis of the size-distribution of price changes. ${ }^{2}$ In particular, we assume that at an exogenous rate $\lambda$ a firm receives an opportunity to adjust its price at no cost, as in a Calvo setup. The model has four fundamental parameters: the size of the menu cost relative to curvature of the profit function $\psi / B$, the volatility of idiosyncratic cost shocks $\sigma^{2}$, the number of products $n$ and the arrival rate of free adjustments $\lambda$.

The model yields four new theoretical results. First we characterize how the inaction set behaves as a function of the parameters. For a small menu cost $\psi / B$ the model behaves as in Barro (1972), Dixit (1991) and Golosov and Lucas (2007): the size of the inaction set displays the usual high sensitivity (i.e. a "quartic root") with respect to the cost and the volatility of the shocks $\sigma^{2}$ (the option value effect). Interestingly, the decision rule is unaffected by the presence of the free adjustments as long as the menu cost is small. The decision rule changes substantially for large menu costs, an assumption that is useful to generate behavior that approaches that of a Calvo model. In this case the size of the inaction set changes with the square root of the menu cost and the arrival rate, and somewhat surprisingly it becomes unresponsive to the volatility of idiosyncratic shock $\sigma^{2}$, so that changes in the uncertainty faced by firms induce no change in behavior (i.e. there is no option value). This result can be used to analyze the effects of "uncertainty shocks" which have received a lot of attention recently, as in e.g. Vavra (2013). Second, the model provides a mapping between the cost of price adjustment and the model parameters: we give a complete analytical characterization of the menu cost implied by observable statistics such as the frequency and the variance of price changes. This mapping can be used to quantify a value of $\psi / B$ consistent with the

\footnotetext{
${ }^{2}$ Absent random menu cost the multi-product model can at most produce a zero excess kurtosis.
} 
evidence on the costs of price adjustment, as measured by e.g. Levy et al. (1997). It thus provides a new dimension to assess the plausibility of previous models, such as Calvo pricing, by inspecting the magnitude of their implied adjustment costs.

Third, by aggregating the optimal decision rules across firms we characterize the frequency $N\left(\Delta p_{i}\right)$, standard deviation $\operatorname{Std}\left(\Delta p_{i}\right)$, and shape of the distribution of the price changes, e.g. its kurtosis: $\operatorname{Kur}\left(\Delta p_{i}\right)$. We show that for any pair of parameters $\{n, \lambda\}$, the two remaining parameters $\{\psi / B, \sigma\}$ map one-to-one onto the observables $N\left(\Delta p_{i}\right), \operatorname{Std}\left(\Delta p_{i}\right)$. This mapping is convenient for the analysis because it allows us to "freeze" the two observables $N\left(\Delta p_{i}\right)$ and $\operatorname{Std}\left(\Delta p_{i}\right)$, which one can take from the data, while retaining the flexibility to accommodate various shapes for the size-distribution of price changes as well as various data on the cost of price adjustment. In particular, we show that the shape of the distribution of price changes can be written exclusively in terms of $n$ and the fraction of free-adjustments $\ell \equiv \lambda / N\left(\Delta p_{i}\right)$. To summarize, the parameterization of the model can be thought as follows: the parameters $\psi / B$ and $\sigma$ are pinned down by the observations on $N\left(\Delta p_{i}\right), \operatorname{Std}\left(\Delta p_{i}\right)$, while the parameters $(n, \ell)$ are pinned down by the shape of the distribution of price changes, e.g. by its kurtosis, and by the data on the costs of price adjustment. In our model the shape of the distribution of price changes ranges from bimodal (for the model where $\ell=0$ and $n=1$ as in the GolosovLucas model) to Normal (for $n=\infty$ and $\ell=0$, our version of Taylor or Reis (2006)'s model), and Laplace (in the case $\ell=1$ for any $n$, our version of the Calvo model). In those three models the kurtosis of price changes is, respectively, 1, 3 and 6 . For any given $\ell$ the level of kurtosis is increasing in $n$. Likewise, for a given $n$ the level of kurtosis is increasing in $\ell^{3}{ }^{3}$

Fourth, we use the model to characterize analytically the impulse responses of the aggregate economy to a once-and-for-all unexpected permanent increase of the money supply. The aggregate effect of a monetary shock depends on the shock size, the frequency, and the kurtosis of price adjustments. The dependence on the size of the shock is a hallmark of menu cost models: monetary shocks that are large (relative to the size of price adjustments) lead to almost all firms adjusting prices and hence imply neutrality. We provide a detailed analysis of the minimum size of the shock that delivers this neutrality (full price flexibility). Instead, small shocks yield real output effects whose size crucially depends on $N\left(\Delta p_{i}\right)$ as well as on the kurtosis of price changes, $\operatorname{Kur}\left(\Delta p_{i}\right)$, a result that is new in the literature and was discussed in equation (1). ${ }^{4}$

Although the model assumes a zero steady-state inflation, we show analytically that a

\footnotetext{
${ }^{3}$ In our set-up a given kurtosis may be obtained by different combinations of $n$ and $\ell$, yet we argue that models with high $n$ yield a better representation of the cross-sectional data because it eliminates the predominant mass of large price changes that arises in models where $n$ is small.

${ }^{4}$ Furthermore, our decomposition of the determinants of the real output effect shows how to measure and aggregate across heterogenous sectors, see Appendix R.
} 
small inflation has only a second order effect on both the kurtosis of the price changes, the frequency of price changes as well as on the cumulative real output effect. Hence the model can be applied to economies with a positive (but small) inflation rate, as observed in most industrialized economies.

\section{Other related literature}

In addition to the papers cited above, our analysis relates to a large literature on the propagation of monetary shocks in sticky price models, unifying earlier results that compare the propagation in the Calvo model with the propagation in either the Taylor or the menu cost model of sticky prices. ${ }^{5}$ We show that introducing a random adjustment costs serves a similar role as that of fat-tailed shocks in Midrigan (2011), increasing the real effect of monetary shocks and bringing the model behavior closer to a Calvo model (yet there are some differences, see the concluding remarks in Section 5 for a discussion).

Our model is related to CalvoPlus model of Nakamura and Steinsson (2010) who consider firms facing idiosyncratic cost shocks as well as a menu cost that oscillates randomly between a high and a small value. In their model, like in ours, the random menu cost makes the adjustment decision state dependent, a feature that dampens substantially the real effect of monetary shocks relative to the Calvo model. Our paper also relates to the analysis of Dotsey, King, and Wolman (1999) who study numerically the propagation of shocks when firms face a menu cost that is drawn from a distribution with a smooth density, but face no idiosyncratic shocks and more recently the work by Vavra (2013). ${ }^{6}$ Given the numerical nature of these contributions, these papers do not provide an explicit map between the model fundamentals, the steady state statistics and the propagation of shocks. We see our results as complementary to theirs. Our model allows for an analytical characterization of the firm's decision rule, the economy's steady state statistics, the identification of the key model parameters, as well as a characterization of the relationship between these parameters/statistics and the size of the output effect of monetary shocks.

The paper is organized as follows: the next section presents the cross section evidence on price setting behavior for France and the US. Section 3 presents the theoretical model and its cross section predictions: it is shown that the model has fundamentally four parameters and

\footnotetext{
${ }^{5}$ A selected list is Chari, Kehoe, and McGrattan (2000), Kiley (2002), Caballero and Engel (2007), Golosov and Lucas (2007). Chari, Kehoe, and McGrattan (2000) and Kiley (2002) obtain that, controlling for the frequency of price changes, the response of output is stronger and more persistent under Calvo than under Taylor contracts. Golosov and Lucas (2007) compare a monetary shock in a menu cost and a Calvo model, with similar frequencies of price change, and find that the half-life of the response to the shock in the Calvo set-up is about five times larger than in the menu cost model.

${ }^{6}$ Dotsey, King, and Wolman (2009) extend their previous contribution of a random menu cost model to incorporate idiosyncratic shocks.
} 
we discuss the mapping between those and observable measures of price setting behavior. Section 4 derives the model predictions on the effect of an unexpected monetary shock. Section 5 discusses the robustness and scope of our main results.

\section{The distribution of price changes: micro-evidence}

A vast amount of research has investigated the patterns of price changes at the microeconomic level in the past decade. A recurring fact that emerges from those studies is that the size distribution of price changes exhibits a large amount of small price changes, as noted by Klenow and Malin (2010); Cavallo (2010); Klenow and Kryvtsov (2008); Chen et al. (2008) and Midrigan $(2009,2011)$ using selected samples of micro data from the US as well as many other industrial countries. This section revisits this evidence using a detailed dataset of price quotes underlying the French Consumer Price Index (about 65\% of the CPI weights from 2003 to 2011). We discuss measurement error by comparing the CPI data with other sources, presumably less affected by measurement error. Finally, we compare our evidence with comparable evidence for the US.

Two issues that are investigated in detail concern heterogeneity and measurement error. Heterogeneity across type of goods and of outlets is pervasive in price data. A well known result is that a mixture of distributions with different variances and the same kurtosis will have a larger kurtosis. For this reason we standardize the data at levels at which we suspect there is heterogeneity in the variances and focus on the kurtosis of the pooled data. We define the standardized price changes, $z$, by demeaning and dividing by the standard deviation of price changes at fine cell levels. A cell is a category of good and of outlet type. We then compute the statistics for the pooled standardized data. ${ }^{7}$ The nature of the correction for measurement error is to compare the CPI statistics with data for similar goods and outlet types that are less affected by measurement error, as in the internet store scraped data from Cavallo (2010), and the scanner data sets used by Midrigan (2011), Eichenbaum et al. (2014) among others. We also analyze the effect of outliers by looking at the differential effect of trimming across datasets. The practice of normalizing the data as well as removing outliers has been used before, as in e.g. Klenow and Kryvtsov (2008); Midrigan (2009).

We find it useful to compare the empirical distribution of price changes to three parametric distributions ordered in terms of increasing frequency of "extreme" price changes: the binomial, the Normal, and the Laplace distribution. Our analysis shows that, after removing the (time invariant) cross section heterogeneity and correcting for measurement error, the size distribution of price changes still features a considerable mass of large as well as small

\footnotetext{
${ }^{7}$ The model of Section 3 allows for sector (and/or good or outlet) heterogeneity and discusses aggregation.
} 
price changes, relative to the binomial distribution implied by the standard menu cost model. Overall we conclude that, after taking into account heterogeneity and measurement error, the shape of the empirical distribution of price changes lays in between a Normal and a Laplace distribution. To quantify the presence of extreme price changes we focus on statistics that are informative about the shape of the size distribution, that are appropriate for symmetric, zero-mean, distributions and that are scale-free. These statistics measure the frequency of extreme (i.e. large and small) observations relative to the standard deviation of the distribution. Because of its prominent role in our theoretical analysis we will focus especially on kurtosis whose value, for the benchmark Binomial, Normal and Laplace distribution, is 1, 3 and 6 respectively.

\subsection{The French microeconomic data underlying the CPI}

The data are a longitudinal dataset of monthly price quotes collected by the INSEE in order to compute the French CPI, over the period 2003:4 to 2011:4. ${ }^{8}$ Each record relates to a precisely defined product sold in a particular outlet in a given month. It contains the price level of the product, as well as limited additional information such as an outlet identifier, an index (when relevant) for package size (say 1 liter) and flags indicating the presence of sales. The raw dataset contains around 11 million price quotes and covers about $65 \%$ of the CPI weights. ${ }^{9}$ The dataset also includes CPI weights, which we use to compute aggregate statistics. Price changes are computed as 100 times the log-difference in prices per unit. To minimize the presence of measurement errors we discarded observations with item substitutions (which might give rise to spurious price changes) and removed "outliers" which, in our baseline analysis, we defined as price changes whose absolute value is smaller than 0.1 percent, or larger than (about) 120 percent. $^{10}$

An important issue with the data on price changes is the treatment of sales. The relevance of dealing with sales in analyzing price stickiness was emphasized by Nakamura and Steinsson (2008); Kehoe and Midrigan (2007) and Midrigan (2011) inter alia. The INSEE dataset contains an indicator variable that identifies whether a given observed price corresponds to a sales promotion discount (either seasonal sale or temporary discounts). ${ }^{11}$ Price changes that

\footnotetext{
${ }^{8}$ The dataset is documented in details in Berardi, Gautier, and Le Bihan (2013).

${ }^{9}$ Some categories of goods and services are not available in our sample: fresh foods, rents, and prices centrally collected by the statistical institute - among which car prices and administered and public utility prices (e.g. electricity). Note that, while rents are out of our dataset, cost of owner-occupied housing is not incorporated in the French CPI, so the share of housing in the CPI is lower than in some other countries.

${ }^{10}$ Some sales involve large discounts, up to $70 \%$, e.g. in clothing. The upper threshold $100 \cdot \log (10 / 3)$, which is about 120 percent log points, allows us to accommodate the after-sale price to return to the original level. See Appendix B for more information and several robustness checks.

${ }^{11}$ The flag is documented by the field agent rather than constructed using a statistical filter. Baudry et al.
} 
Figure 1: Histogram of Standardized Price Adjustments: French CPI 2003-2011

All data

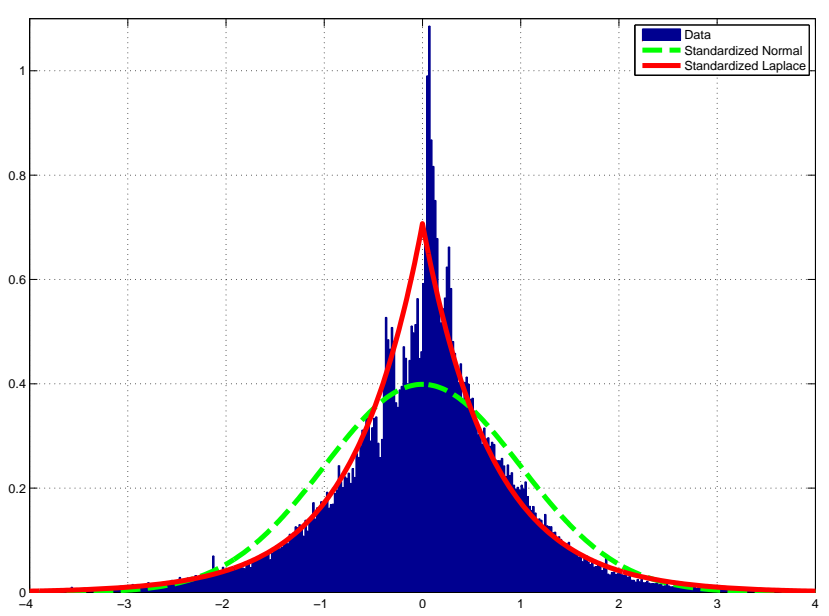

Excluding sales

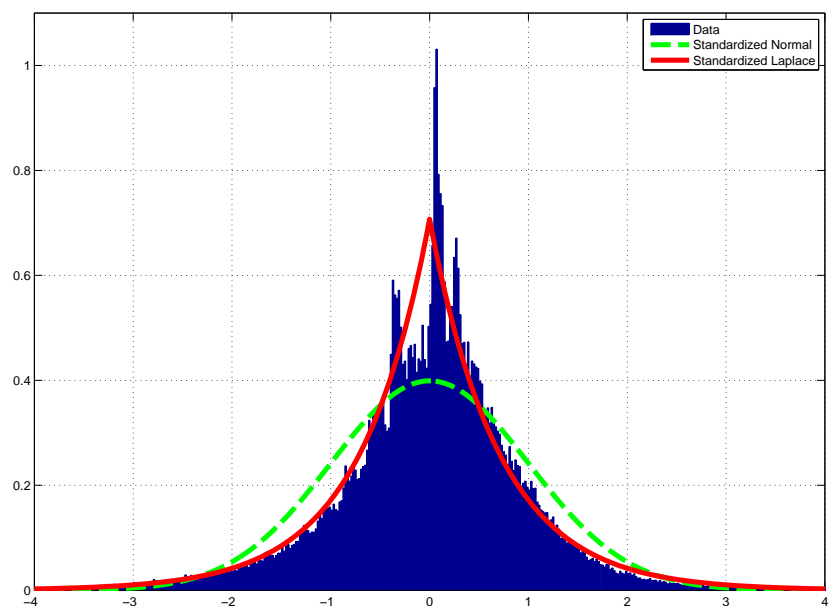

The figures use the elementary CPI data from France (2003-2011). Price changes are the log difference in price per unit, standardized by good category (272) and outlet type (11) and pooled. Price changes equal to zero are discarded. The upper panel uses about 1.5 million data points, the lower panel about 1.1 million.

result from sales (including price changes from a sales price to a regular one) account for approximately $17 \%$ of all the price changes. Overall, the incidence of sales on the frequency of price change is less important than in the US where according to Nakamura and Steinsson (2008) the share of price change due to sales is $21.5 \%$. In the following, as a robustness check, we report results both with and without sales observations.

We now document the patterns on the peakedness and thick tails of the distribution of price changes. As those patterns vary considerably across sectors and outlet types, a concern already mentioned is that a large variance and kurtosis of price changes may essentially reflect that observations of price changes are drawn from a mixture of distributions, and thus may be artefacts. We consider a breakdown of the data into $J$ "cells", where each cell is defined by a good category and outlet type (for instance, one cell will be bread in supermarkets). We will here use the finest partition possible in our data (each cell is a COICOP category at the 6-digit level in an outlet type) and have around 1,500 cells. ${ }^{12}$ In each cell $j$ the standardized price change at date $t$ for good $i$ is defined as $z_{j i t}=\left(\Delta p_{j i t}-m_{j}\right) / \sigma_{j}$ where $m_{j}$ and $\sigma_{j}$ are the mean and standard deviation of price changes in cell $j$, and price changes equal to zero are disregarded (index $i$ reflects that there is typically more than one store or good type per cell

(2007) investigate the extent of "undetected" sales and conclude this is a limited concern.

${ }^{12}$ There are 11 outlet types and 272 CPI categories. Not every category of good is sold in every outlet type so there are about half of the potential 2,992 cells. 
e.g., different types of bread or different stores selling bread in the sample at a given date).

Figure 1 is a weighted histogram of the standardized price changes. On the same graph we superimpose the density of the standard Normal distribution as well as the standardized Laplace distribution (both have unit variance). The Laplace distribution has a kurtosis of 6 and is thus more peaked than the Normal. It is apparent that the empirical distribution of standardized price changes is closer to the Laplace distribution than to the Normal. ${ }^{13}$ We also consider the statistic $\mathbb{E}\left[\left|\Delta p_{i}\right|\right] / \operatorname{Std}\left(\Delta p_{i}\right)$ as a (reverse) measure of the frequency of extreme price changes. The main difference of this statistic with respect to kurtosis is that it is less sensitive to extreme outliers. For the Binomial, Normal and Laplace distributions the reference values for this statistic are: $1,0.80$ and 0.70 .

Table 1 reports the frequency of price changes as well as selected moments of the distribution of price changes. The frequency of price change is around $17 \%$ per month, or about 2 price changes per year. The fraction of price decreases among price changes is around $40 \%$. The average absolute price change (not reported in the table) is sizable $(9.2 \%)$, as is the standard deviation of price changes (16.6\%). These patterns match those documented by Alvarez et al. (2006) for the Euro area. With the qualification that the frequency of price changes is typically found to be smaller in the Euro area than in the US, they also broadly match the US evidence by e.g. Nakamura and Steinsson (2008). The kurtosis and peakedness of the distribution of price changes have not been quantitatively documented so far on European data. The kurtosis of non-standardized price changes is very high: 12.8. This level of kurtosis is of same order of magnitude as that documented by Klenow and Malin (2010) for the US. As argued above, a high kurtosis is likely the consequence of a mixture of observations taken from distributions with different variances. Considering the standardized price changes, i.e. correcting for cross-section heterogeneity in the variances, reduces kurtosis to 8.9 (also, kurtosis is halved on data excluding sales). Unfortunately the information available in the CPI data prevents us from correcting heterogeneity at a finer level (e.g., we do not know the UPC of the product or the store where it is sold). In other databases where more information is available the reduction in the measured kurtosis is even more prominent, as shown using US scanner dataset in Appendix B.1. It is shown that kurtosis falls by another 30 to $40 \%$ when moving to the product-store level.

The fraction of extreme (small or large) price changes is noticeable. The fraction of absolute standardized price changes lower than one fourth of the mean is 22.2 percent. Also 12.9 percent of absolute normalized price changes are larger than 2 times the mean of the absolute standardized price change. Overall, it appears that these figures are very close to the

\footnotetext{
${ }^{13}$ In Appendix B.2 we provide similar histograms by groups of good at a disaggregated level. Most of them have the same pattern as Figure 1, that is a distribution that is more peaked than the gaussian, and often more peaked than the Laplace.
} 
Table 1: Selected moments from the distribution of price changes

\begin{tabular}{|c|c|c|c|c|}
\hline & \multicolumn{2}{|c|}{ Data } & \multicolumn{2}{|c|}{ Benchmarks } \\
\hline & \multicolumn{2}{|c|}{ all records exc.sales } & \multicolumn{2}{|c|}{ Normal Laplace } \\
\hline Frequency of price changes & 17.1 & 14.7 & & \\
\hline Fraction of price changes that are decreases & 39.2 & 35.7 & & \\
\hline \multicolumn{5}{|c|}{ Moments for the size of price changes: $\Delta p_{i}$} \\
\hline Average & 0.3 & 1.1 & & \\
\hline Standard deviation & 16.6 & 8.0 & & \\
\hline Kurtosis & 12.8 & 20.9 & & \\
\hline \multicolumn{3}{|c|}{ Moments of standardized price changes: $z$} & \multirow[b]{2}{*}{3} & \multirow[b]{2}{*}{6} \\
\hline Kurtosis & 8.89 & 10.40 & & \\
\hline \multicolumn{3}{|c|}{ Moments for the absolute value of standardized price changes: $|z|$} & & \\
\hline Average: $\mathbb{E}(|z|)$ & 0.70 & 0.69 & 0.80 & 0.70 \\
\hline Fraction of observations $<0.25 \cdot \mathbb{E}(|z|)$ & 22.2 & 20.7 & 15.8 & 22.1 \\
\hline Fraction of observations $<0.5 \cdot \mathbb{E}(|z|)$ & 39.3 & 38.6 & 31.0 & 39.4 \\
\hline Fraction of observations $>2 \cdot \mathbb{E}(|z|)$ & 12.9 & 12.5 & 11.1 & 13.5 \\
\hline Fraction of observations $>4 \cdot \mathbb{E}(|z|)$ & 1.8 & 2.0 & 0.0 & 1.8 \\
\hline Number of obs. with $\Delta p_{i} \neq 0$ & $1,544,829$ & $1,080,183$ & & \\
\hline
\end{tabular}

Source is INSEE, monthly price records from French CPI, data from 2003:4 to 2011:4. Coverage is around $65 \%$ of CPI weight since rents, and prices of fresh food and centrally collected items (e.g. electricity, train and airplane tickets) are not included in the dataset. Frequency of price change is the average fraction of price changes per month, in percent. Size of price change is the first-difference in the logarithm of price per unit, expressed in percent. Observations with imputed prices or quality change are discarded. Observations outside the interval $0.1 \leq\left|\Delta p_{i}\right| \leq 100 \cdot \log (10 / 3)$ are removed as outliers. "Exc. sales" exclude observations flagged as sales by the INSEE data collectors. Moments are computed aggregating all prices changes using CPI weights at the product level. The third and fourth panels report moments for the standardized price change $z_{i j t}=\frac{\Delta p_{i j t}-m_{j}}{\sigma_{j}}$ where $m_{j}$ and $\sigma_{j}$ are the mean and standard deviation of price changes in category $j$ (see the text). The Normal and Laplace distributions used in the last two columns have a zero mean and standard deviation equal to one.

ones that would be produced by a (standardized) Laplace distribution. Consistently, the size of the average absolute standardized price change in the data is equal to 0.70 , the same value that obtains for the statistic $\mathbb{E}\left[\left|\Delta p_{i}\right|\right] / \operatorname{Std}\left(\Delta p_{i}\right)$ if $\Delta p_{i}$ follows a Laplace distribution. There is a mass of large price changes, say larger than 4 standard deviations, that are virtually absent in the Gaussian case.

Removing sales has a large effect on the variance of price changes, as indicated by the results reported in the second column of Table $1 .{ }^{14}$ However, removing sales does not affect our findings on the peakedness of the distribution. Kurtosis actually increases when sales

\footnotetext{
${ }^{14}$ We remove the observations flagged as sales as well as the subsequent increase back to the "regular" price. To compute the standardized non-sales price changes, we first discard sales-related observations, then standardize the data.
} 
observations are removed both in the raw data as well as in the standardized data. This is also visible in the right panel of Figure 1 which plots the distribution of standardized (ex-sales) price changes.

\subsection{Measurement error and the estimation of kurtosis}

In this section we discuss evidence on the magnitude of both very large and very small price changes due to measurement error, and discuss its effect on measures of kurtosis. Eichenbaum et al. (2014) warned that the small price changes recorded in the data may reflect measurement error. Appendix B.3 explores the concerns raised by Eichenbaum et al. (2014) and concludes that they also apply to the French data, albeit to a lesser extent. We analyze here the consequences of one particular type of measurement error and derive a simple correction for kurtosis. We show that a small amount of this measurement error, inconsequential for measuring the aggregate the cost of living, may have sizeable consequences for the measurement of the descriptive statistics displayed in Table 1, such as kurtosis, and suggest a procedure to correct for it.

Let the observed price changes $\Delta p_{m}$ be given by a mixture of two distributions:

$$
\Delta p_{m}= \begin{cases}\Delta p_{u} & \text { with prob. } \zeta \\ \epsilon & \text { with prob. } 1-\zeta\end{cases}
$$

where we interpret $\epsilon$ as measurement error and $\Delta p_{u}$ as a "true" price change. This assumption aims to capture that, even at the finest level of disaggregation, some price changes in the CPI data are the consequence of small product substitutions (e.g. different brands for a given good being recorded) which do not reflect an actual change in the good's price. Likewise, in scanner dataset, spuriously small price changes may originate from the weekly nature of the prices being recorded, which e.g. averages customers with and without discount coupons. Assume the distribution of $\Delta p_{u}$ has standard deviation $\sigma_{u}$ and kurtosis $k_{u}$. Likewise the distribution of $\epsilon$ has standard deviation $\sigma_{e}$ and kurtosis $k_{e}$. Both distributions are assumed to have zero expected value. One interpretation is that quality changes (not recorded by the statistical office) generate "artificial" price changes. We assume that these price changes are small, i.e. that $\sigma_{e}$ is small, and that the process for the unreported change in quality is independent of the "true" changes in prices. The kurtosis of the observed price changes, $\operatorname{Kur}\left(\Delta p_{m}\right)$, is then equal to: $\operatorname{Kur}\left(\Delta p_{m}\right)=k_{u} \frac{\zeta \sigma_{u}^{4}+\left(k_{\epsilon} / k_{u}\right) \sigma_{e}^{4}}{\zeta^{2} \sigma_{u}^{4}+(1-\zeta)^{2} \sigma_{e}^{4}+2 \zeta(1-\zeta) \sigma_{e}^{2} \sigma_{u}^{2}}$. Letting $\sigma_{e}$ go to zero we obtain that kurtosis measured over the (observed) price changes is: $\lim _{\sigma_{e} \downarrow 0} \operatorname{Kur}\left(\Delta p_{m}\right)=k_{u} / \zeta$. Thus, if the sample includes a fraction $\zeta$ of true price changes and the rest are spuriously imputed small price changes the kurtosis will increase by a factor $1 / \zeta$, relative to the kurtosis of the 
true distribution. ${ }^{15}$ The limit $\operatorname{Kur}\left(\Delta p_{m}\right)=k_{u} / \zeta$ suggests to quantify $\zeta$ by comparing the observed kurtosis across a sample with measurement error and one without. We now turn to addressing this issue empirically.

We match a subset of our French CPI data with the prices for several French retailers taken from the Billion Price Project (BPP) dataset (see Cavallo (2010)). The BPP data are "scraped" on-line, thus they are arguably less contaminated by measurement errors. ${ }^{16}$ We compare the results obtained using the scraped BPP data from two large retailers with our results based on the CPI data for a similar type of outlet: to this end we restrict our dataset to CPI price records in "hypermarkets", excluding gasoline. We also compare with the BPP data from a large French retailer specialized in electronic and appliances. In that case we restrict the CPI dataset to goods in the category of appliances and electronic using the Coicop nomenclature, collected in outlets type "hypermarkets", "supermarkets", and "large area specialists". Comparing the values of kurtosis from both data sets suggests that $\zeta \cong 0.5$. We can apply this magnitude to the full sample of CPI data of Table 1 , for which no "measurement error-free" counterpart like the BPP exists, to obtain a corrected kurtosis. The number thus obtained for the kurtosis ranges between 4 and 5 (using the kurtosis of 8.9 of standardized price changes), so it lays in between the kurtosis of the Normal and the Laplace distribution.

Sensitivity to trimming. To assess the hypothesis that large price changes are due to measurement error we compare the differential effect on kurtosis of trimming large (absolute value) price changes in CPI vs trimming them in scanner data. For the French CPI we find that excluding (log percent) price changes for which $\left|\Delta p_{i}\right| \geq 100 \log (2)$, instead of just excluding those that are $\left|\Delta p_{i}\right| \geq 100 \log (10 / 3)$, decreases kurtosis from 8.9 to 7.2 (see lines 4 and 8 of Table 6 ). We interpret this as evidence of measurement error under the hypothesis that large price changes in the CPI are due to transcription data errors, which are virtually absent in internet scraped data. ${ }^{17}$ Similar evidence on the effects of trimming using US scanner data is presented in the next section.

\footnotetext{
${ }^{15}$ Under this interpretation the number of measured price changes, denoted by $N_{a m}$ will be higher than the number of true price changes per unit of time, say $N_{a u}$. Let's denote $N_{a \epsilon}$ the expected number of incorrectly imputed price changes. We have: $N_{a m}=N_{a u}+N_{a \epsilon}=\zeta N_{a m}+(1-\zeta) N_{a m}$. Thus if we have two estimates of $\operatorname{Kur}\left(\Delta p_{i}\right)$ and of $N\left(\Delta p_{i}\right)$ and we assume that one has no measurement error and the other has a fraction $\zeta$ of small imputed price changes as described above, can estimate $\zeta$ using either the ratio of the two estimates of kurtosis or the ratio of the two estimates of the number of price changes per unit of time.

${ }^{16}$ We are extremely grateful to Alberto Cavallo for sharing part of his data with us.

${ }^{17}$ In the French CPI we have observed cases where a single large price change which reverts to its original value, which corresponds to consecutive prices that are equal except for the transposition of a digit, which is almost surely a clerical error.
} 
Table 2: Comparison of the CPI vs. the BPP data in France

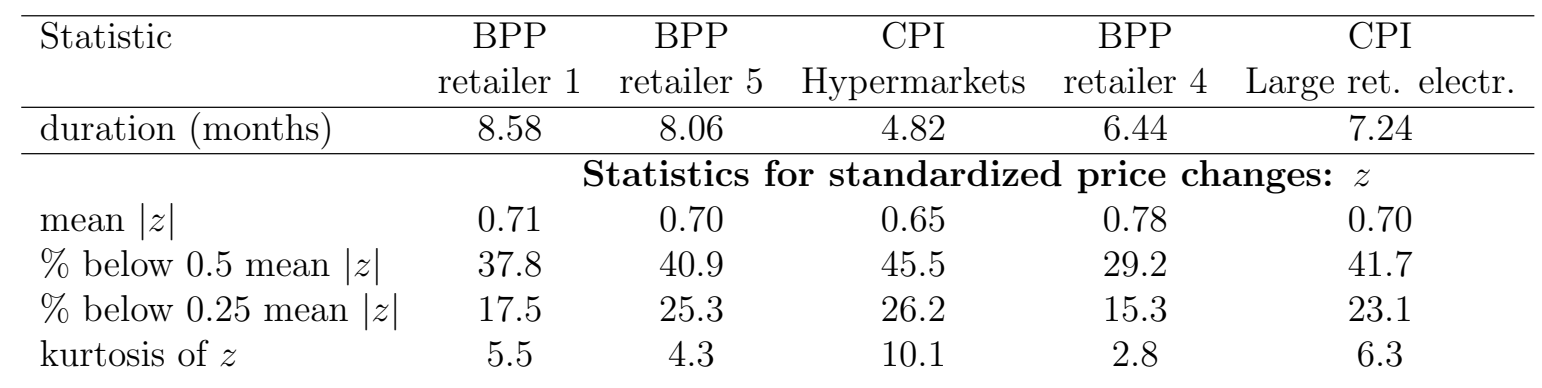

Note: The BPP data are documented in Cavallo (2010). Results were communicated by the author. For CPI data source is INSEE, monthly price records from French CPI, data from 2003:4 to 2011:4. Sub-sample in column (3) is price records in outlet type "hypermarkets". Sub-sample in column (5) is goods in the category of appliances and electronic , as identified using the Coicop nomenclature, collected in the following outlets type: "hypermarkets", "supermarkets", and "large area specialists". Data are standardized within each subsample using Coicop categories.

\subsection{A comparison with the US data}

This section compares the French evidence with the US evidence presented, respectively, in Klenow and Kryvtsov (2008) and in the scanner data used by Eichenbaum, Jaimovich, and Rebelo (2011), as well as other scanner datasets. Figure 2 plots four histograms: two are price changes from the US and French CPI data, while the other two are theoretical benchmarks. The first one (in red) is the distribution of standardized (weighted) price changes (excluding sales) for the US based on Figure 3 of Klenow and Kryvtsov (2008). ${ }^{18}$ Since the distribution is truncated at -3 and +3 , its standard deviation is 0.83 instead of 1 . Its kurtosis is 6.95 . The second histogram (in blue) is the distribution of the standardized price changes (excl. sales) for the French CPI, constructed using the trimming criteria used for the US. This distribution has a standard deviation 0.95 and a kurtosis of 4.42. The smaller standard deviation and much smaller kurtosis than in Table 1 are due to the discretization and truncation. To see the effect of these treatment of the data, note that Klenow and Malin (2010) report a kurtosis of 10 for posted prices and 17.4 for regular prices, without discretizing, censoring, or standardizing the data. For comparison Vavra (2013) finds that after trimming the data in a way similar to our treatment of the French data, but without standardizing it, the kurtosis of US CPI price changes is 6.4. On the other hand, Vavra finds that the kurtosis of standardized price changes is 4.9. ${ }^{19}$ The figure also reports the standardized Normal and Laplace distri-

\footnotetext{
${ }^{18}$ The histogram has twenty four bins, spaced every 0.25 units, of the distribution of standardized regular price changes (excl. sales). The standardization was done by ELI, the narrowest categories of goods. After standardization the distributions are weighed according to the CPI weight.

${ }^{19}$ See Table IV and footnote 6 of Vavra (2013) for the specifics on the trimming. We thank Vavra for providing this statistics which is not available in his paper.
} 
butions (discretized and truncated). The main message of Figure 2 is that the histogram of standardized, non-sales, price change are rather similar in France and the US. Furthermore, in both cases the shape is closer to that of a Laplace distribution than to a Gaussian one (and consistently with previous sub-section, in both cases we conjecture measurement error explains why these distributions are actually more peaked than the Laplace).

Figure 2: Histogram of Standardized Price Adjustments: US and French CPI

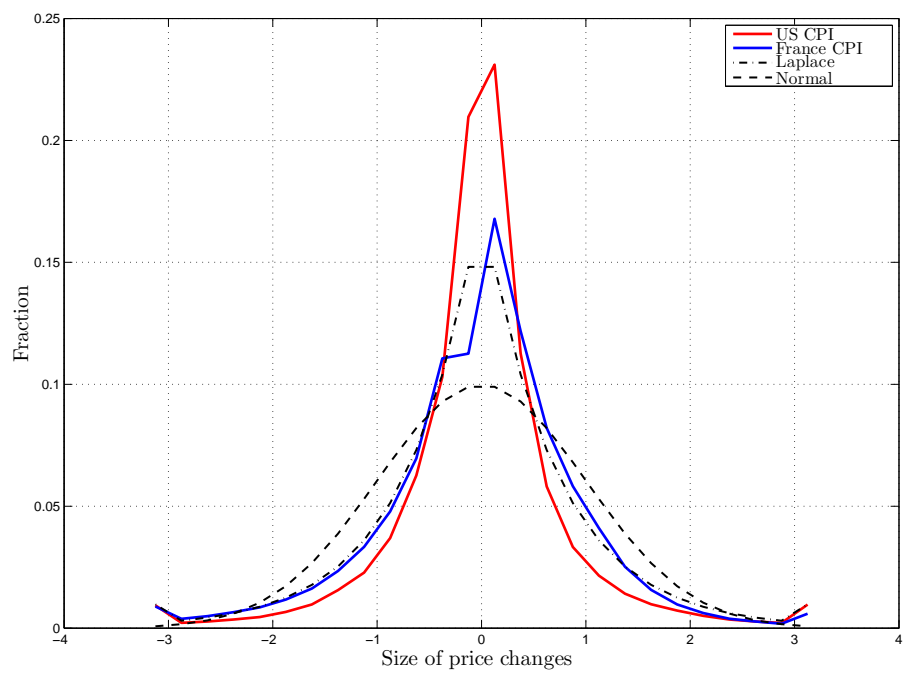

Sales data are excluded. Data for France are from the CPI as in Figure 1. The CPI data for the US are taken from Figure 3 in Klenow and Kryvtsov (2008). Price changes equal to zero are discarded.

Table 3 provides a further comparison based on datasets presumably less subject to measurement errors. For France we use data from the BPP, and those from hypermarkets in the CPI dataset. For the US we use the scanner data evidence reported by Eichenbaum, Jaimovich, and Rebelo (2011), Midrigan (2011), as well as our own analysis of the SymphonyIRI scanner dataset. As reflected by the summary statistics, the distribution is somewhat more peaked in France; for instance the kurtosis is 5 in the BPP against values between 3 and 4 in US scanner datasets described below. Overall, we find that the share of small price changes is non-negligible in both countries. For completeness we report complementary evidence taken from scanner data sets, described in more detail in the online Appendix B.1. While generally immune to measurement errors due to transcription and product substitutions, even scanner price data are affected by measurement error since they report average weekly prices for an item, so that observed prices are an average of prices paid by customers with and without discounts. We hypothesize, as Midrigan (2011) and Eichenbaum et al. 
(2014) among others, that the weekly averaging introduces spurious small price changes. The EJR column of Table 3 uses the data from Eichenbaum, Jaimovich, and Rebelo (2011): after standardizing at the good-store level and removing very extreme price changes (smaller than $0.1 \%$ or larger than $120 \%$ (log points) as in our benchmark for France), yields a kurtosis of 3.0. Alternatively, trimming price changes smaller than one dollar cent, certainly due to measurement error, the kurtosis falls little below the one of the Normal. Likewise, using the larger Symphony-IRI dataset (see Bronnenberg, Kruger, and Mela (2008)), shows that removing extreme price changes and standardizing the data reduces kurtosis from a level of about 30 to 4.3. Finally, we notice that the effect of extreme price changes on the measurement of kurtosis is also present in the CPI. For the French CPI excluding (log percent) price changes for which $\left|\Delta p_{i}\right| \leq 1$ as opposed to only excluding those price changes for which $\left|\Delta p_{i}\right| \leq 1 / 10$, reduces kurtosis from 7.2 to 6.3 (see lines 4 vs 11 of Table 6 ). For the Norvegian CPI Wulfsberg (2010) finds that kurtosis is also sensitive to both large and small price changes, removing the 1 and 99 percentiles decreases kurtosis from 8.1 to 5.7.

Table 3: Comparison across datasets for large Hypermarkets in France and the US

\begin{tabular}{lcc|ccc}
\hline & \multicolumn{2}{c|}{ France } & \multicolumn{3}{c}{ US (scanner data) } \\
& CPI & BPP & EJR & Symphony IRI Midrigan (2009) \\
\hline \multirow{2}{*}{ mean of $|z|$} & 0.65 & 0.70 & 0.78 & 0.73 & - \\
\% below 0.50 mean $|z|$ & 45 & 39 & 33 & 39 & 29 \\
\% below 0.25 mean $|z|$ & 24 & 21 & 23 & 25 & 13 \\
kurtosis of $z$ & 10 & 5 & 3.0 & 4.3 & 3.5 \\
\hline \hline
\end{tabular}

$\overline{\text { All price changes including sales. The BPP statistics for France are an average of the ones reported in Table }} 2$. The EJR scanner data are from Eichenbaum, Jaimovich, and Rebelo (2011), the IRI scanner data are from the Symphony-IRI database described in Appendix B.1. For both scanner data sets individual observations are average weekly prices. We remove price changes outside the interval $0.1 \leq\left|\Delta p_{i}\right| \leq 100 \cdot \log (10 / 3)$ and standardize the data at the good (upc) store level (see Appendix B.1). The data from Midrigan (2009) are taken from his Table 1 and 2b, using simple averages of the AC Nielsen and Dominick's scanner data. Midrigan standardizes price changes by dividing by the mean absolute value of the price changes at the product-store-month level. He also removes price changes smaller than 1 cent or larger than $100 \%$.

Overall we conclude that, after accounting for heterogeneity and measurement error, the presence of both small and large price changes appears relevant in France as well as in the US. The shape of the standardized empirical distribution of price changes lays in between a Normal and a Laplace distribution. The distribution appears close to a Normal in the US and closer to Laplace in France. We summarize the results of this section by stating that the standardized distribution of price changes, after taking measurement error and heterogeneity into account, has kurtosis of about 4 for US and of about 5 for France. 


\section{A tractable menu cost model}

This section presents a menu cost model aimed at qualitatively matching the patterns documented above. In the canonical menu cost model price adjustments occur when a threshold is hit, so that the implied distribution of price changes fails to generate the small changes that appear in the data (see the discussion in Midrigan (2011); Cavallo (2010); Alvarez and Lippi (2014)). The model that we propose here is able to produce a large mass of small price changes and the positive excess kurtosis that we documented above. Two ingredients are key to this end: (i) the random menu costs and that (ii) the menu cost faced by the firm, $\psi$, applies to a bundle of $n$ goods, so that after paying the fixed cost the firm can reprice one or all goods at no extra cost. Each of these assumptions individually is capable to generate some small price changes and higher kurtosis than in a canonical model where $n=1$ and where menu costs are constant. The assumption of random menu costs is key to generate a positive excess kurtosis in the distribution of price changes. The combination of the two is important: in the models where $n=1$ (with or without random menu costs) the distribution of price changes has a mass point at the adjustment threshold, a feature that is in stark contrast with the evidence discussed above. The prominence of "large" price changes (i.e. a "U shaped" distribution) persists even in a model with $n=2$, as in Midrigan (2011) where the distribution of price changes asymptotes near the adjustment threshold, or $n=3$ as in Bhattarai and Schoenle (2014). We show below that in order to generate a shape of the size distribution that is comparable to the one in the data one needs $n \geq 6$.

\subsection{A random menu cost problem for a firm selling $n=1$ good.}

Consider a firm whose profit-maximizing price at time $t, p^{*}(t)$, follows the process $\mathrm{d} p^{*}(t)=$ $\sigma \mathrm{d} W(t)$ where $W(t)$ is a standard brownian motion with no drift, and $\sigma$ is the standard deviation of the iid innovations to $p^{*}$. The technology to change prices is as follows: to change the price at will the firm needs to incur a fixed menu cost of size $\psi$. However, with some probability the firm receives an opportunity to adjust the price "for free". Assume this probability is Poisson, i.e. that the free-adjustments have a constant hazard rate per unit of time, equal to $\lambda$. Let $p(t)$ denote the "price gap" at time $t$, i.e. the difference between the actual sale price $P(t)$ and the profit maximizing price $p^{*}(t)$, i.e. $p(t) \equiv P(t)-p^{*}(t)$. The instantaneous firm losses (i.e. reduction in profits) created by the price gap are given by the quadratic function: $B p^{2}(t)$. Let $V(p)$ be the present-value cost function for a firm with price gap $p$. Upon the arrival of a free adjustment opportunity the firm optimally resets the price 
gap to zero, hence the Bellman equation for the range of inaction reads:

$$
r V(p)=B p^{2}+\lambda[V(0)-V(p)]+\frac{\sigma^{2}}{2} V^{\prime \prime}(p), \quad \text { for } p \in(-\bar{p}, \bar{p})
$$

where $\bar{p}$ is the threshold rule defining the region where inaction is optimal (see Appendix O for the calculations of this section). This equation states that the flow value of the Bellman equation is given by the instantaneous losses, $B p^{2}$, plus the expected change in the value function, which is due either to a free adjustment (with rate $\lambda$ in which case the price gap is reset to zero) or to the volatility of shocks $\sigma^{2}$ (there is no first order derivative of the value function since the price gaps have no drift). The value-matching and smooth-pasting conditions are given by $V(\bar{p})=V(0)+\psi$ and $V^{\prime}(\bar{p})=0$. Two properties of the optimal threshold $\bar{p}$ are worth noticing (proved later for any $n$ ): the value function, and the optimal decision rules, are a function of $\lambda+r$, as opposed to each of them separately. Intuitively this is because when a free adjustment opportunity occurs the price gap is adjusted, so that $\lambda$ acts as an addition to the discount factor. Second, for a small value of $\psi / B$ or a small value of $\lambda+r$, the value of $\bar{p}$ is insensitive to $\lambda+r$. More precisely, the derivative of $\bar{p}$ with respect to $\lambda+r$ is zero as $\psi / B$ or $\lambda+r$ tend to zero. A Taylor expansion of the value function yields the following approximate optimal threshold $\bar{p}=\left(\frac{6 \psi \sigma^{2}}{B}\right)^{\frac{1}{4}}$ which is accurate when $\psi / B$ is small. ${ }^{20}$

Computing the expected time between adjustments yields an expression for the average number of adjustments per period, $N\left(\Delta p_{i}\right)$, which we use to measure the fraction of free adjustments over the total number of adjustments, a variable we call $\ell$, as

$$
\ell \equiv \frac{\lambda}{N\left(\Delta p_{i}\right)}=\frac{e^{\sqrt{2 \phi}}+e^{-\sqrt{2 \phi}}-2}{e^{\sqrt{2 \phi}}+e^{-\sqrt{2 \phi}}} \in(0,1) \quad \text { where we define } \quad \phi \equiv \frac{\lambda \bar{p}^{2}}{\sigma^{2}}
$$

which shows that the fraction of free adjustments $\ell$ depends only on the parameter $\phi$. The parameter $\phi$ can be interpreted as the ratio between $\lambda$, the number of free adjustments, and $\sigma^{2} / \bar{p}^{2}$, the number of adjustments in a model where $\lambda=0$ and the threshold policy $\bar{p}$ is followed.

The distribution of price changes $w\left(\Delta p_{i}\right)$ is symmetric around $\Delta p_{i}=0$. This distribution has a mass point at $\Delta p_{i}= \pm \bar{p}$ with probability $1-\ell$, i.e. this is the fraction of price changes that occurs because the price gap reaches the boundaries of the inaction region. The remaining fraction of price changes, $\ell$, occurs when a free adjustment opportunity arrives, at which time the price gap is set to zero. Price changes in the range $p \in(-\bar{p}, \bar{p})$ have a density

20 Exactly the same expression was established by Barro (1972) and Dixit (1991) for the case in which $\lambda=0$. Below we discuss an approximate threshold for the case in which $\psi$ is large. 
$\ell g(p)$ where $g(p)$ denotes the density of the invariant distribution of price gaps given by

$$
g(p)=\frac{\sqrt{2 \phi}}{2 \bar{p}\left(e^{\sqrt{2 \phi}}-1\right)^{2}}\left(e^{\sqrt{2 \phi}\left(2-\frac{|p|}{\bar{p}}\right)}-e^{\sqrt{2 \phi} \frac{|p|}{\bar{p}}}\right) \quad \text { for } \quad p \in[-\bar{p}, \bar{p}] .
$$

Thus the distribution of price changes is given by

$$
\left\{\begin{array}{lll}
\operatorname{Pr}\left(\Delta p_{i}=-\bar{p}\right)=\operatorname{Pr}\left(\Delta p_{i}=\bar{p}\right) & =\frac{1}{2}(1-\ell) & \\
\operatorname{Pr}\left(\Delta p_{i} \in d p\right) & =\ell g(p) d p \equiv w\left(\Delta p_{i}\right) d p & \text { for } p \in(-\bar{p}, \bar{p})
\end{array}\right.
$$

which is a symmetric "tent shaped" distribution in the $(-\bar{p}, \bar{p})$ interval with the two mass points at the boundaries $\pm \bar{p}$. As detailed below the kurtosis of this distribution is increasing in $\lambda$, and in particular the distribution of price changes is more peaked than that of a standard menu cost model where $\lambda=0$.

We make two remarks about this simple model which will hold, and be generalized, in the more general model developed next. The first one is that the shape of the distribution of price changes depends only on the fraction of free adjustments $\ell$ (or, equivalently, on $\phi$ ). This means that two economies, or sectors, that differ in the standard deviation of price changes $\operatorname{Std}\left(\Delta p_{i}\right)$ and/or in the frequency of price adjustment $N\left(\Delta p_{i}\right)$ will display a distribution of price changes with exactly the same shape (once its scale is adjusted) provided that they have the same value of $\ell$. This property is useful to aggregate the sectors of an economy that are heterogenous in their steady state features $N\left(\Delta p_{i}\right), \operatorname{Std}\left(\Delta p_{i}\right)$. Because of this property the ratio of moments from the size distribution of price changes, such as kurtosis, are scale free and can be used to retrieve information on $\phi$. The second property, which we state here and prove below for the more general economy, is that the "shape" of the impulse response function of this economy to a (once and for all) monetary shock depends only on $\ell$. We will show how one can simply scale (or relabel) one or both axes of an impulse function to analyze economies with the same $\ell$ that differ in either $N\left(\Delta p_{i}\right)$ or $\operatorname{Std}\left(\Delta p_{i}\right)$.

\subsection{Extending the model to multi-product firms}

This section incorporates the model with free adjustment opportunities discussed above into the model of Alvarez and Lippi (2014) where the firm is selling $n$ goods, so that $p$ is now a vector in $\mathbb{R}^{n}$, but pays a single fixed adjustment cost to change the $n$ prices. We incorporate this feature for several reasons. First, as explained above, in the model with $n=1$ good there is a mass point on price changes of size $\left|\Delta p_{i}\right|=\bar{p}$. For $n=2$ the mass point disappears but the distribution of price changes still features the largest mass of observations (the highest density) near the adjustment thresholds. There is no evidence of this in any data set we can 
find. Values of $n \geq 3$ eliminate this problem. Second, the model with $\lambda=0$ has a kurtosis that increases with $n$, hence providing an alternative to random menu costs. Third, for large $n$ and $\lambda=0$ the distribution of price changes tends to the Normal distribution, which is both a nice benchmark and an accurate description of the price changes for some sectors. Finally, the multi-product model with $(n>1)$ has an alternative, broader, "rational inattentiveness" interpretation for the adjustment cost $\psi$. In particular, one can assume that the firm freely observes its total profits but not the individual ones (for each product), unless it either pays the cost $\psi$ or a free observation opportunity arrives, in which case it is able to set the optimal price to each of them. This is useful because it allows a broader interpretation of the menu cost, including not only the physical cost of changing prices but also the cost related to gathering and processing the information for individual products. ${ }^{21}$

We now briefly describe the setup of the firm problem with $n$ products. As before the free adjustment opportunities are independent of the driving processes $\left\{W_{i}(t)\right\}$ for price gaps, and arrive according to a Poisson process with constant intensity $\lambda$. In between price adjustments each of the price gaps evolves according to a Brownian motion $\mathrm{d} p_{i}(t)=\sigma \mathrm{d} W_{i}(t)$. It is assumed that all price gaps are subject to the same variance $\sigma^{2}$ and that the innovations are independent across price gaps. ${ }^{22}$

We assume that, when the opportunity arrives, the firm can adjust all prices without paying the cost $\psi$. The analysis of the multi product problem can be greatly simplified by using the sum of the squared price gaps, $y \equiv\|p\|^{2}$ as a state variable, instead of the vector $p=\left(p_{1}, \ldots, p_{n}\right)$, as done in Alvarez and Lippi (2014). The scalar $y$ summarizes the state because the period objective function can be written as a function of it and because, from an application of Ito's lemma, one can derive a one dimensional diffusion which describes its law of motion, namely

$$
\mathrm{d} y=n \sigma^{2} \mathrm{~d} t+2 \sigma \sqrt{y} \mathrm{~d} W
$$

where $W$ is a standard Brownian motion.

Using $N\left(\Delta p_{i}\right)$ and $\operatorname{Var}\left(\Delta p_{i}\right)$ to denote the frequency and the (cross sectional) variance of the price changes of product $i$ the next proposition establishes a useful relationship that holds in a large class of models for any policy for price changes, which we describe by a stopping time rule:

\footnotetext{
${ }^{21}$ As an example, see Chakrabarti and Scholnick (2007) who argue that for stores as Amazon or Barnes and Noble physical menu cost are small, yet prices change infrequently, and thus conclude that the cost may be of a different nature. Interestingly, they find that for such retailers price changes are synchronized across products, which is an implication of the multi-product model.

${ }^{22}$ Alvarez and Lippi (2014) discuss the case with correlated price gaps. Intuitively, as correlation increase the model becomes more similar to the $n=1$ case, since the price changes of a firm become more similar.
} 
Proposition 1 Let $\tau$ describe the time at which a price change takes place, so that all price gaps are closed. Assume the stopping time treats each of the $n$ price gaps symmetrically. For any finite stopping time $\tau$ we have:

$$
N\left(\Delta p_{i}\right) \cdot \operatorname{Var}\left(\Delta p_{i}\right)=\sigma^{2}
$$

The proposition highlights the trade-off for the firm's policy: more frequent adjustments are required to have smaller price gaps. We underline that equation (3) holds for any stopping rule, not just for the optimal one. See Appendix A for the proof, where the reader can verify that the key assumptions are the random walks and symmetry; indeed equation (3) holds for a larger class of models, for instance those with correlated price gaps and a richer class of random adjustment cost.

Upon the arrival of a free adjustment opportunity the firm will set the price gap to zero, hence the Bellman equation for the range of inaction reads:

$$
r v(y)=B y+\lambda[v(0)-v(y)]+n \sigma^{2} v^{\prime}(y)+2 \sigma^{2} y v^{\prime \prime}(y), \quad \text { for } y \in(0, \bar{y}),
$$

where $B y$ is the sum of the deviation from the optimal profits from the $n$ goods.

We note that given the symmetry of the problem after an adjustment of the $n$ prices the firm will set each of the price gap to zero, i.e. will set $\|p\|^{2}=y=0$. The value matching condition is then $v(0)+\psi=v(\bar{y})$, which uses that when $y$ reaches a critical value, denoted by $\bar{y}$, by paying the fixed cost $\psi$ the firm can change the $n$ prices. The smooth pasting condition is $v^{\prime}(\bar{y})=0$.

The next lemma establishes how to solve for $\bar{y}$ using the solution of a simpler problem where $\lambda=0$ discussed in Alvarez and Lippi (2014). It turns out that a simple change of variables allows us to use the solution for the case of $\lambda=0$ to compute the solution for the case of interest in this paper. The change of variables consists in using $r+\lambda$ as the interest rate in the solution of the problem with $\lambda=0$. We have:

Lemma 1 Let $v(y ; r, \lambda)$ and $\bar{y}(r, \lambda)$ be the optimal value function and adjustment threshold for a problem with discount rate $r$ and arrival rate $\lambda$. Then $v(y ; r, \lambda)=v(y ; r+\lambda, 0)+$ $\frac{\lambda}{r} v(0 ; r+\lambda, 0)$ for all $y \geq 0$ and thus $\bar{y}(r, \lambda)=\bar{y}(r+\lambda, 0)$.

The proof of this lemma follows immediately from a guess and verify strategy. The lemma allows us to use the characterization of $\bar{y}$ with respect to $r$ given in Proposition 4 of Alvarez and Lippi (2014) to study the effect of $r+\lambda$ on $\bar{y}$. In Appendix $\mathrm{F}$ we write the analytical solution for the value function, and give more details on it. The next proposition summarizes that result and extends the characterization of the optimal threshold to the case where $\psi$ 
is large, a case that is useful to understand the behavior of an economy with a lot of free adjustments opportunity as in a Calvo mechanism (see Appendix A for the proof).

Proposition 2 Assume $\sigma^{2}>0, n \geq 1, \lambda+r>0$ and $B>0$, and let $\bar{y}$ be the threshold for the optimal decision rule. We then have that:

1. As $\psi \rightarrow 0$ then $\frac{\bar{y}}{\sqrt{2(n+2) \sigma^{2} \frac{\psi}{B}}} \rightarrow 1$ or $\bar{y} \approx \sqrt{2(n+2) \sigma^{2} \frac{\psi}{B}}$.

2. As $\psi \rightarrow \infty$ we have $\frac{\bar{y}}{\psi} \rightarrow(r+\lambda) / B$ or $\bar{y} \approx \frac{\psi}{B}(r+\lambda)$. Moreover this also holds for large $n$ and large $\frac{\psi}{n}$, namely $\lim _{\psi / n \rightarrow \infty} \lim _{n \rightarrow \infty} \frac{\bar{y} / n}{\psi / n}=(r+\lambda) / B$ or $\frac{\bar{y}}{n} \approx \frac{\psi / n}{B}(r+\lambda)$.

The proposition shows that $\bar{y}$ is approximately constant with respect to $\lambda$ for small values of $\psi$, so that for small menu costs the result is the well known quartic root formula (recall that $y$ has the units of a squared price gap) and the inaction region is increasing in the variance of the shock, due to the higher option value. Interestingly, and novel in the literature, the second part of the proposition shows that for large values of the adjustment cost the rule becomes a square root and that the optimal threshold does not depends on $\sigma$, which shows that for large adjustment costs the option value component of the decision becomes negligible. Moreover, when the menu costs are large the threshold $\bar{y}$ is increasing in $\lambda$ : the prospect of receiving a free adjustment tomorrow increases inaction today.

We now turn to the discussion of the model implications for the frequency of price changes. We let $N\left(\Delta p_{i}\right)$ be the expected number of adjustments per unit of time of a model with a given $\lambda$ and $\bar{y}$. We establish the following (see Appendix A for the proof):

Proposition 3 Let $\Gamma$ denote the gamma function. The fraction of free adjustments is $\ell=$ $\lambda / N\left(\Delta p_{i}\right)$, where

$$
\ell=\frac{\sum_{i=1}^{\infty} \frac{\Gamma\left(\frac{n}{2}\right)}{i ! \Gamma\left(\frac{n}{2}+i\right)}\left[\frac{n}{2}\right]^{i} \phi^{i}}{\sum_{i=0}^{\infty} \frac{\Gamma\left(\frac{n}{2}\right)}{i ! \Gamma\left(\frac{n}{2}+i\right)}\left[\frac{n}{2}\right]^{i} \phi^{i}} \equiv \mathcal{L}(\phi, n) \text {, where } \phi \equiv \frac{\lambda \bar{y}}{n \sigma^{2}}
$$

The proposition shows that $\ell$ is a function only of two variables: $n$ and $\phi$, and that it is increasing in $\phi$. As was the case for the model with $n=1$, the parameter $\phi$ can be interpreted as the ratio between $\lambda$, the number of free adjustments, and $n \sigma^{2} / \bar{p}^{2}$, the number of adjustments in a model where $\lambda=0$ and the threshold policy $\bar{y}$ is followed. For a given $n$ there is a one to one and onto mapping between $\phi$ and $\ell$ : as $\phi \rightarrow 0$ then $\ell \rightarrow 0$, and as $\phi \rightarrow \infty$ then $\ell \rightarrow 1$.

We now turn to characterizing the invariant distribution of $y$ for the case where $\lambda>0$, a key ingredient to compute the size-distribution of price changes. The density of the invariant 
distribution solves the Kolmogorov forward equation: $\frac{\lambda}{2 \sigma^{2}} f(y)=f^{\prime \prime}(y) y-\left(\frac{n}{2}-2\right) f^{\prime}(y)$ for $y \in(0, \bar{y})$, with the two boundary conditions $f(\bar{y})=0$ and $\int_{0}^{\bar{y}} f(y) d y=1$. It is clear from these conditions that $f(\cdot)$ is uniquely defined for a given triplet: $\bar{y}>0, n \geq 1$ and $\lambda / \sigma^{2} \geq 0$. The general solution of this ODE is

$$
f(y)=\left(\frac{\lambda y}{2 \sigma^{2}}\right)^{\left(\frac{n}{4}-\frac{1}{2}\right)}\left[C_{1} I_{\nu}\left(2 \sqrt{\frac{\lambda y}{2 \sigma^{2}}}\right)+C_{2} K_{\nu}\left(2 \sqrt{\frac{\lambda y}{2 \sigma^{2}}}\right)\right]
$$

where $I_{\nu}$ and $K_{\nu}$ are the modified Bessel functions of the first and second kind, $C_{1}, C_{2}$ are two arbitrary constants and $\nu=\left|\frac{n}{2}-1\right|$, see Zaitsev and Polyanin (2003) for a proof. The constants $C_{1}, C_{2}$ are chosen to satisfy the two boundary conditions. ${ }^{23}$ While the density in equation (6) depends on 3 constants $n, \phi$ and $\bar{y}$, its shape depends only on 2 constants, namely $n$ and $\phi$, as formally stated in Lemma 2 in Appendix A. The lemma shows that one can normalize $\bar{y}$ to 1 and compute the density for the corresponding $\phi$. Finally we also use equation (6) to find a closed form expression for $f$ in terms of a power series displayed in equation (32), derived in Appendix C.

We denote the marginal distribution of price changes by $w\left(\Delta p_{i}\right)$. Recall that a firm changes all prices when $y$ first reaches $\bar{y}$ or when a free adjustment opportunity occurs even though $y<\bar{y}$. Therefore to characterize the price changes $\Delta p_{i}$ of good $i$ belonging to the vector of price gaps $p$ we need three objects: the fraction of free adjustments $\ell$, the invariant distribution $f(y)$ and the marginal distribution of price changes conditional on a value of $y$, $\omega\left(\Delta p_{i} ; y\right)$ which, following Proposition 6 of Alvarez and Lippi (2014) when $n \geq 2$, is

$$
\omega\left(\Delta p_{i} ; y\right)= \begin{cases}\frac{1}{\operatorname{Beta}\left(\frac{n-1}{2}, \frac{1}{2}\right) \sqrt{y}}\left(1-\left(\frac{\Delta p_{i}}{\sqrt{y}}\right)^{2}\right)^{(n-3) / 2} & \text { if }\left(\Delta p_{i}\right)^{2} \leq y \\ 0 & \text { if }\left(\Delta p_{i}\right)^{2}>y\end{cases}
$$

where $\operatorname{Beta}(\cdot, \cdot)$ denotes the Beta function. In this case the (cross-sectional) standard deviation of the price changes is $\operatorname{Std}\left(\Delta p_{i} ; y\right)=\sqrt{y / n}$. The marginal distribution of price changes $w\left(\Delta p_{i}\right)$ is given by

$$
w\left(\Delta p_{i}\right)=\omega\left(\Delta p_{i} ; \bar{y}\right)(1-\ell)+\left[\int_{0}^{\bar{y}} \omega\left(\Delta p_{i} ; y\right) f(y) d y\right] \ell \quad \text { for } n \geq 2 .
$$

For the case when $n=2$ the density of the price changes diverges at the boundaries of the domain where $\Delta p_{i}= \pm \sqrt{\bar{y} / n}$, as can be seen in Figure 3. This feature echoes the two mass

${ }^{23}$ We note that both modified Bessel functions are positive, that $I_{\nu}(y)$ is exponentially increasing with $I_{\nu}(0) \geq 0$, and that $K_{\nu}(y)$ is exponentially decreasing with $K_{\nu}(0)=+\infty$. 
points that occur in the $n=1$ case where a non-zero mass of price changes occurs exactly at the boundaries. For $n \geq 6$ the shape of the density takes a tent-shape, similar to the one that is seen in the data. As the fraction of free adjustments approaches 1 the shape of the density function converges to the shape of the Laplace distribution. The next proposition shows that $n$ and $\ell$ completely determine the shape of the distribution of price changes (see Appendix A for the proof):

Proposition 4 Let $w\left(\Delta p_{i} ; n, \ell, 1\right)$ be the density function for the price changes $\Delta p_{i}$ in an economy with $n$ goods, a share $\ell$ of free adjustments, and a unit standard deviation of price changes $\operatorname{Std}\left(\Delta p_{i}\right)=1$. This density function is homogenous of degree -1 in $\Delta p_{i}$ and $\operatorname{Std}\left(\Delta p_{i}\right)$, which implies

$$
w\left(a \Delta p_{i} ; n, \ell, a\right)=\frac{1}{a} w\left(\Delta p_{i} ; n, \ell, 1\right) \quad \text { for all } \quad a>0
$$

The proposition establishes that the "shape" of the size distribution of price changes has 2 parameters: $n$ and $\ell$. Every two economies sharing these parameters will have the same size distribution of price changes once the scale is adjusted. The proposition implies that we can aggregate firms or industries that are heterogenous in terms of frequency $N\left(\Delta p_{i}\right)$ and standard deviation of price changes $\operatorname{Std}\left(\Delta p_{i}\right)$ provided that $n$ and $\ell$ are the same. Notice in particular that the frequency of price changes $N\left(\Delta p_{i}\right)$ does not have an independent effect on the distribution of price changes as long as $\ell$ remains constant.

Figure 3: Size distribution of price changes
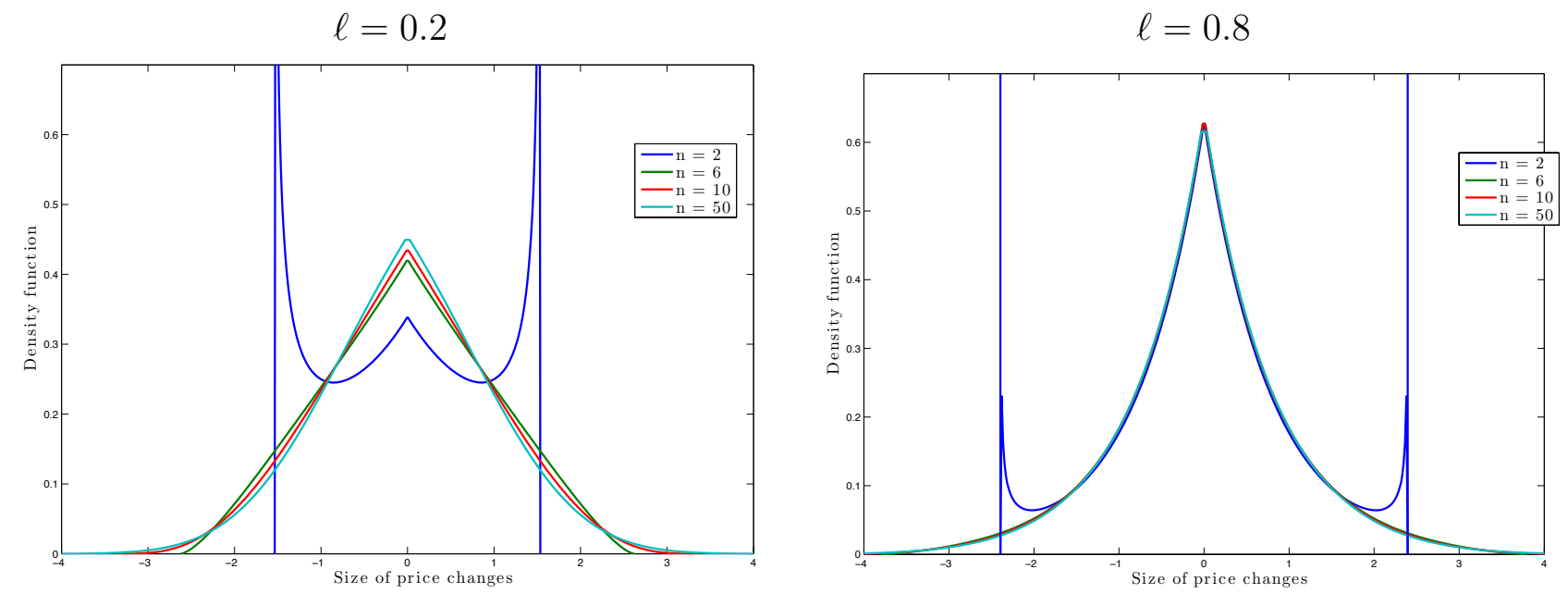

Note: All distributions are zero mean with unit standard deviation. As stated in Proposition 4 the shape of this distribution only depends on $\ell$ and $n$. 
Notice that the distribution $w\left(\Delta p_{i}\right)$ is a mixture of the $\omega\left(\Delta p_{i}, y\right)$ densities. These densities are scaled versions of each other with different standard deviations. This increases the kurtosis of the distribution of price changes compared to the case where $\lambda=0$. In particular Proposition 6 in Alvarez and Lippi (2014) shows that the variance and kurtosis of $\omega\left(\Delta p_{i}, y\right)$ are given by $y / n$ and $3 n /(n+2)$ respectively. Using that $\Delta p_{i}$ is distributed as a mixture of the $\omega\left(\Delta p_{i}, y\right)$, we can compute several moments of interest, such as

$$
\begin{aligned}
\operatorname{Var}\left(\Delta p_{i}\right) & =(1-\ell) \frac{\bar{y}}{n}+\ell \int_{0}^{\bar{y}} \frac{y}{n} f(y) d y \\
\operatorname{Kur}\left(\Delta p_{i}\right) & =\frac{3 n}{2+n} \frac{(1-\ell) \bar{y}^{2}+\ell \int_{0}^{\bar{y}} y^{2} f(y) d y}{\left[(1-\ell) \bar{y}+\ell \int_{0}^{\bar{y}} y f(y) d y\right]^{2}}>\frac{3 n}{2+n}
\end{aligned}
$$

It is immediate from Proposition 4 that the value of the kurtosis $\operatorname{Kur}\left(\Delta p_{i}\right)$ depends only on two parameters: $n$ and $\ell$. For instance, if one were to change the parameters $\psi / B, \lambda$ and $\sigma^{2}$ keeping the same values for $\ell$ and $n$, the kurtosis of the price changes would not change. Moreover, kurtosis is increasing in both $n$ and $\ell$, as can be seen in Figure 5 which plots the value of $\operatorname{Kur}\left(\Delta p_{i}\right) / 6$ for various combinations of $n$ and $\ell$, the only two parameters determining kurtosis. For small values of $\ell$ kurtosis is increasing in $n$ up to a level of 3 . For instance, if $\ell=0$ and $n \rightarrow \infty$, the kurtosis converges to 3 since the distribution of price changes at the time of adjusting for each firm becomes normal; this value is the highest that the purely multi-product model with $\ell=0$ can produce. For any $n$, as the fraction of free adjustments $\ell$ increases, the kurtosis increases towards 6 , the maximum value achieved in our model when $\ell=1 .{ }^{24}$ The inequality that appears in the second line is a well known result: the mixture of distributions with the same kurtosis but with different variances has higher kurtosis, which follows from Jensen's inequality. Equation (33) in Appendix D displays a closed form solution for kurtosis which does not involve solving for $f(y)$.

To conclude the description of the model we summarize a few special cases nested by our setup. The Golosov-Lucas model is obtained when $n=1$ and $\ell=0$, implying a kurtosis of 1. The Taylor model, or equivalently "rational inattentiveness" model by Reis, is obtained when $n=\infty$ and $\ell=0$, implying a kurtosis of 3. The Calvo model is obtained for $\ell \rightarrow 1$, for all values of $n$, implying a kurtosis of 6 . Additionally, two recent models can be proxied: the "CalvoPlus" model by Nakamura and Steinsson (2010) for the special case of no intermediate goods ( $s_{m}=0$ using their notation), is obtained assuming $n=1$ and $\ell \in(0,1)$. The multiproduct model of Midrigan (2011) is obtained assuming $n=2$ and modeling the fat

\footnotetext{
${ }^{24}$ The relationship between the fraction of free adjustment and the "mass of small price changes", a statistic that is closely related to kurtosis, was noticed in the numerical simulations of Nakamura and Steinsson (2010) (see their footnote 15, where their "frequency of low repricing opportunities", $1-\alpha$, is essentially our $\ell$ ).
} 
tailed shock by assuming $\ell \in(0,1) .{ }^{25}$ Depending on the parameter choice for $\ell$, the last two models can generate a kurtosis between 1 and 6. It is shown in Section 4.1 that higher values of kurtosis are essential in both models to explain why the real effects in those models are closer to Calvo than to Golosov-Lucas.

The map between the fundamental parameters and observables. Our model has four independent parameters: the scaled menu cost $\psi / B$, the volatility of shocks $\sigma$, the number of goods $n$ and the rate of free adjustment opportunities $\lambda$. We find it convenient to pin down two of these parameters, namely $\psi / B$ and $\sigma$ by matching observable statistics that are available in micro datasets: the frequency $N\left(\Delta p_{i}\right)$ and the variance $\operatorname{Var}\left(\Delta p_{i}\right)$ of price changes. Given these statistics the model has two residual parameters: $n$ and $\lambda$, with the latter mapping one-to-one and onto $\ell=\lambda / N\left(\Delta p_{i}\right)$. The parametrization of the model can thus be usefully interpreted as choosing $n$ and $\ell$ to match two empirical observations, in addition to $N\left(\Delta p_{i}\right), \operatorname{Var}\left(\Delta p_{i}\right)$. It was shown in Proposition 4 how $\ell$ and $n$ shape the distribution of price changes, in particular its kurtosis. In the next section we show how $\ell$ and $n$ map into the cost of price adjustments, for given values of $N\left(\Delta p_{i}\right)$ and $\operatorname{Var}\left(\Delta p_{i}\right)$. Kurtosis and the costs of price adjustments can thus be used to discipline the parameterization of the model, as we discuss next.

\subsection{On the implied cost of price adjustment}

In this section we give a characterization of the model implications for the size of the menu cost, i.e. a mapping between observable statistics and the value of $\psi / B$ or $\psi$ (we also discuss how to measure $B$ ). We consider two measures for the cost of price adjustment: the first one is the cost of a single price adjustment as a fraction of profits: $\psi / n$. Recall that $\psi$ is the cost that a firm must pay if it decides to adjust all prices instantaneously (i.e. without waiting for a free adjustment). Measuring this cost as a fraction of profits transforms these magnitudes into units that have an intuitive interpretation. The second measure is the average flow cost of price adjustment given by: $N\left(\Delta p_{i}\right) \frac{\psi}{n}(1-\ell)$. This cost measures the average amount of resources that the firm pays to adjust prices per period. The difference between the two measures should be clear: when all price adjustments are costly, as in a model where $\ell=0$, the relevant measure of price adjustments is $\psi / n$, so that the total flow cost of price adjustment borne by a firm per year is $N\left(\Delta p_{i}\right) \psi / n$. Allowing for a fraction of adjustments to be free the total flow cost must be multiplied by $1-\ell$, as some of the adjustments that occur during the period are free. The latter measure is useful because it relates more directly to

\footnotetext{
${ }^{25}$ Yet it differs in that the fat-tailed shocks mainly contribute to large price changes, see Appendix $\mathrm{H}$ for a formal discussion.
} 
what has been measured in the data by Levy et al. (1997); Zbaracki et al. (2004), namely the "average" cost of a price adjustment. The next proposition analyzes the mapping between the scaled menu cost $\psi / n$, and $B, \ell, n, N\left(\Delta p_{i}\right)$ and $\operatorname{Var}\left(\Delta p_{i}\right)$.

Proposition 5 Fix the number of products $n \geq 1$ and let $r \downarrow 0$. There is a unique triplet $\left(\sigma^{2}, \lambda, \psi\right)$ consistent with any triplet $\ell \in[0,1], \operatorname{Var}\left(\Delta p_{i}\right)>0$ and $N\left(\Delta p_{i}\right)>0$. Moreover, fixing any value $\ell$, the menu cost $\psi \geq 0$ can be written as:

$$
\frac{\psi}{n}=B \frac{\operatorname{Var}\left(\Delta p_{i}\right)}{N\left(\Delta p_{i}\right)} \Psi(n, \ell)
$$

where $\Psi$ is only a function of $(n, \ell)$. For all $n \geq 1$ the function $\Psi(n, \cdot)$ satisfies:

$$
\begin{aligned}
& \lim _{\ell \rightarrow 0} \Psi(n, \ell)=\frac{n}{2(n+2)}, \quad \lim _{\ell \rightarrow 1} \Psi(n, \ell)=\infty, \lim _{\ell \rightarrow 1} \Psi(n, \ell)(1-\ell)=0, \\
& \lim _{\ell \rightarrow 1} \frac{\Psi\left(n^{\prime}, \ell\right) / n^{\prime}}{\Psi(n, \ell) / n} \leq 1 \text { for } n^{\prime} \geq n, \text { and } \lim _{n \rightarrow \infty} \frac{\Psi(n, \ell) / n}{\Psi(1, \ell) / 1} \rightarrow 0 \text { as } \ell \rightarrow 1
\end{aligned}
$$

Equation (11) shows that for any fixed $n \geq 1$ and $\ell \in[0,1]$ the menu cost $\psi$ is proportional to the ratio $\operatorname{Var}\left(\Delta p_{i}\right) / N\left(\Delta p_{i}\right)$. This is intuitive: economies with higher frequency of price changes are obtained by having a proportionally lower menu cost, and economies with more extreme price changes, are obtained with a proportionally higher menu cost. Second, equation (11) shows that the menu cost is proportional to $B$, which measures the benefits of closing a (unit square) price gap. The parameter $B$ is related to the curvature of the profit function, and thus it relates to demand elasticities and mark-ups. Using a fully specified microeconomic problem where firms face a constant demand elasticity $\eta$ (equal across products) gives that $B=\eta(\eta-1) / 2$, which can be written in terms of the (net) markup over marginal costs $\mathfrak{m} \equiv 1 /(\eta-1)$ so that $B=(1+\mathfrak{m}) /\left(2 \mathfrak{m}^{2}\right) .{ }^{26}$ The last expression is useful to calibrate the model using empirical estimates of the markup such as the ones by Christopoulou and Vermeulen (2012): the estimated markups average around $28 \%$ for the US manufacturing sector, and around $36 \%$ for market services (slightly smaller values are obtained for France, see their Table 1). ${ }^{27}$ A similar value for the US, namely a markup rate of about $33 \%$, is used by Nakamura and Steinsson (2010).

The left panel of Figure 4 illustrates the comparative static effect of $\ell$ and $n$ on the implied menu cost, fixing $B \operatorname{Var}\left(\Delta p_{i}\right) / N\left(\Delta p_{i}\right)$, i.e. it plots the function $\Psi(n, \ell)$. Fixing

\footnotetext{
${ }^{26}$ Nakamura and Steinsson (2010) notice that lower markups (higher values of demand elasticity) $\eta$ must imply higher menu costs, as shown by equation (11). Footnote 14 in their paper discusses evidence on the markup rates across several microeconomic studies and macro papers.

27 The evidence for the US services is consistent with the gross margins, based on accounting data, reported in the Annual Retail Trade Survey by the US Census (see http://www.census.gov/retail/).
} 
Figure 4: Implied cost of price adjustment

Cost of one price adjustment $\psi / n$ (as $\%$ of profits)

Yearly cost of adjustment (as \% of revenues)
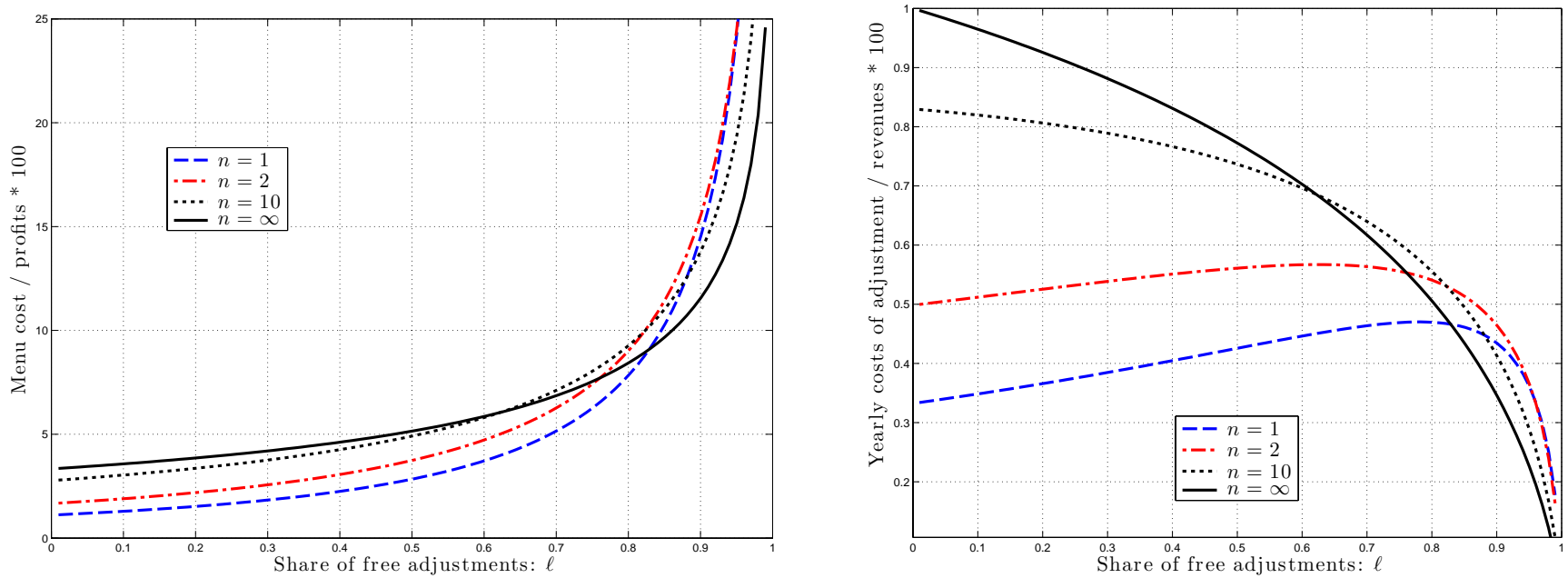

All economies in the figures feature $\operatorname{Std}\left(\Delta p_{i}\right)=0.10$ and a markup of $25 \%$. For those in the left panel we set $N\left(\Delta p_{i}\right)=1.5$.

a value of $n$ it can be seen that the menu cost $\psi / n$ is increasing in $\ell$. This is intuitive: a larger fraction of free adjustments $\ell$ requires a higher menu cost, since firms must choose not to adjust prices even when the current price is far away from their ideal price. Indeed equation (12) shows that as $\ell \rightarrow 1$, the implied menu cost diverges to $+\infty$. This happens because, in our version of Calvo's model, the menu cost must prevent any price change, and since the underlying shocks are assumed to follow a random walk in some instances there are arbitrarily large benefits of changing prices. On the other hand, for $\ell=0$ and $n=1$, our version of Golosov-Lucas's model, the menu cost attains its smallest (strictly positive) value. Fixing $\ell$ and moving across lines shows that the implied fixed cost $\psi / n$ is not monotone in the number of products $n$. Indeed, as stated in equation (12) for a very small share $\ell$ the values of $\psi / n$ are increasing in $n$. On the other hand, for larger value of the share $\ell$, the order of the implied fixed cost is reversed.

The model also has clear predictions about the per period (say yearly) cost of price adjustments borne by the firms: $(1-\ell) N\left(\Delta p_{i}\right) \psi / n$. In spite of the fact that the cost of a single deliberate price adjustment diverges as $\ell \rightarrow 1$, the total yearly cost of adjustment converge to zero continuously. This can be seen in the right panel of Figure 4. A simple transformation gives the yearly cost of price adjustments as a fraction of revenues: $\frac{(1-\ell) N\left(\Delta p_{i}\right) \psi / n}{\eta}$, where the scaling by $\eta$ transforms the units from fraction of profits into fraction of revenues. ${ }^{28}$ This

\footnotetext{
${ }^{28}$ Since $R=\eta \Pi$ where $R$ is revenues per good and $\Pi$ profits per good.
} 
statistic is useful because it has empirical counterparts, studied e.g. by Levy et al. (1997). Using equation (11) and the previous definition for the markup yields

$$
\frac{\text { Yearly costs of price adjustment }}{\text { Yearly revenues }}=\frac{1}{2} \frac{\operatorname{Var}\left(\Delta p_{i}\right)}{\mathfrak{m}}(1-\ell) \Psi(n, \ell)
$$

Figure 4 plots the two cost measures in equation (11) and (14) as functions of $\ell, n$ for an economy with $N\left(\Delta p_{i}\right)=1.5, \operatorname{Std}\left(\Delta p_{i}\right)=0.10$ and a markup $\mathfrak{m} \approx 25 \%$ (i.e. $B=10$ ). We see this parametrization as being consistent with the US data on price adjustments, markups, and the size distribution of price changes discussed above. Alternative parametrizations are readily computed, as discussed below. The figure illustrates how observations on the costs of price adjustments can be used to parametrize the model. Levy et al. (1997) and Dutta et al. (1999) (Table IV and Table 3, respectively) document that for multi-product stores (a handful of supermarkets chain and one drugstore chain) the average cost of price adjustment is around 0.7 percent of revenues. For an economy with $n=10$ (a reasonable parametrization to fit the size-distribution of price changes) the right panel of the figure shows that the model reproduces the yearly cost of $0.7 \%$ of revenues when the fraction of free adjustments $\ell$ is around $60 \%$. The upper panel indicates that at this level of $\ell$ the cost of one price adjustment is around $5 \%$ of profits.

\section{The cumulative output effect of a monetary shock}

In this section we discuss the response of the economy's aggregate output to an unexpected (once and for all) increase of the money supply of size $\delta$, starting from a steady state with zero inflation. Our main objective is to characterize the total cumulative output effect of the monetary shock, namely the area under the output's impulse response, as a function of the 4 fundamental model parameters: the frequency $N\left(\Delta p_{i}\right)$, standard deviation $\operatorname{Std}\left(\Delta p_{i}\right)$, the number of products in the firm's bundle $n$, and the fraction of free adjustments $\ell{ }^{29}$ While the main focus of this section is on the cumulative output effect of a monetary shock, in Appendix $\mathrm{G}$ we also provide a characterization of the whole impulse response of the aggregate price level (and hence of output).

\footnotetext{
${ }^{29}$ Caballero and Engel (2007) perform a related exercise using the Caplin Spulber (S,s) model augmented with a random opportunity of price change, which occurs at rate $\lambda$. They study how increasing $\lambda$ affects the response of the price level (see their Figure 3). There are two channels through which this works: by affecting the frequency of price adjustment and by changing the size and mass of price adjusters. One important difference with respect to them is that our comparative static analysis of a higher $\lambda$ is done keeping $N\left(\Delta p_{i}\right)$ and $\operatorname{Std}\left(\Delta p_{i}\right)$ constant. As discussed at the beginning of Section 3.3 this implies adjusting the parameters $\sigma$ and $\psi / B$ accordingly.
} 
General Equilibrium Setup. The general equilibrium set up is essentially the one in Golosov and Lucas (2007), adapted to multi-product firms as in Alvarez and Lippi (2014) (see their Appendix B). Each firm produces $n$ goods, each with a linear labor only technology, subject to idiosyncratic productivity shocks independent across products, whose logs follows a $\mathrm{BM}$ with instantaneous variance $\sigma^{2}$. As in the previous sections, a firm is subject to a random menu cost to simultaneously change the price of its $n$ products. In a period of length $d t$ this cost equals $\psi_{\ell}$ units of labor with probability $1-\lambda d t$, or zero. Also each firm faces a demand with constant elasticity $\eta>1$ for each of its $n$ products, coming from households's CES utility function for the consumption aggregate. To keep the expenditure shares stationary across goods, in the face of the permanent idiosyncratic shocks, we assume an offsetting preference shocks. The $p_{i}(t)$ in our previous sections are the logs of the markups in each product of the firm relative to the static optimal markup, and our quadratic objective function can be taken to be a second order expansion on the firm's profits with $B=(1 / 2) \eta(\eta-1)$. Households' have a constant discount rate $r$ and an instantaneous utility function which is additively separable: $\log$ in real balances, linear in leisure, and has constant intertemporal elasticity of substitution $1 / \epsilon$ for the consumption aggregate, so that the labor supply elasticity to real wages is $1 / \epsilon$.

Area under output's impulse response. We focus on the cumulative sum (integral) of the output above the steady-state level after a monetary shock of size $\delta>0$. This measure is closely related to the output variance due to monetary shocks, which is sometimes used in the literature. ${ }^{30}$ We define the cumulative output effect $\mathcal{M}$ after a shock $\delta$ as:

$$
\mathcal{M}(\delta)=\frac{1}{\epsilon} \int_{0}^{\infty}(\delta-\mathcal{P}(\delta, t)) d t
$$

where $\mathcal{P}(\delta, t)$ is the cumulative effect of the monetary shock $\delta$ on the $(\log )$ of the aggregate price level $t$ periods after the shock (see Appendix $\mathrm{G}$ for a rigorous definition of $\mathcal{P}(\delta, t)$ and a characterization of the impulse response). Intuitively, the argument of the integral gives the aggregate real wages at time $t$, which are then mapped into output according to the labor supply elasticity $1 / \epsilon$. Integrating over time gives the total cumulative effect.

To characterize $\mathcal{M}(\delta)$ we consider the expected cumulative output deviation from steady state of a firm with a vector of price gaps $p$ :

$$
m\left(p_{1}, \ldots, p_{n}\right)=-\mathbb{E}\left[\int_{0}^{\tau} \sum_{i=1}^{n} p_{i}(t) d t \mid p(0)=p\right]
$$

\footnotetext{
${ }^{30}$ For more discussion and evidence on this equivalence see footnote 21 of Nakamura and Steinsson (2010).
} 
where $\tau$ is the stopping time associated with the optimal decision rule described as the first time that $\|p(t)\|^{2}$ reaches threshold $\bar{y}$ or that a free adjustment opportunity arrives. Note that, by definition, if $\|p\|^{2} \geq \bar{y}$ then $m(p)=0$. Intuitively, a firm with a price gap $-p_{i}(t)$ for good type $i$ is producing $p_{i}(t) / \epsilon$ percent excess output compared to its steady state at time $t$. Thus, integrating over time until the (random) time of adjustment $\tau, m(p) / n$ gives the expected total excess output produced by a firm that immediately after the monetary shock has a vector of price gaps equal to $p$. Three remarks about this solution are in order. First, given the GE structure, identical to the one in Golosov and Lucas (2007), a once and for all increase in money supply is followed by a once and for all increase in nominal wages, and leaves nominal interest rates unaltered. Second, by using the steady state decision rule, $\bar{y}$, we are ignoring the general equilibrium feedback effects. In Proposition 7 of Alvarez and Lippi (2014) we showed that, given a combination of the general equilibrium set-up in Golosov and Lucas (2007) and the lack of the strategic complementarities, these effects are of second order. ${ }^{31}$ Third, we use that after the first price change the expected contribution to output of each firm is zero since positive and negative output contributions are equally likely, i.e. $m(0)=0$. This is convenient since it allows us to characterize the propagation of the monetary shocks without having to keep track of the time evolution for the whole distribution of price gaps.

The function $m(p)$ defined in equation (16) is extremely useful: exploiting the knowledge about the infinitesimal changes of the state, and properties of the period payoffs, yields a full characterization of the cumulative impulse response without having to solve for (or simulate) the whole impulse response function. The idea, which is novel in the macroeconomics literature, follows the same logic used to compute expected values using a Bellman (or Kolmogorov) equation and maps the characterization of $m(p)$ into the solution of a differential equation. For example in the $n=1$ case, where $p$ is a scalar price gap in $(-\bar{p}, \bar{p})$, a Bellmanequation type of logic gives $\lambda m(p)=-p+m^{\prime \prime}(p) \sigma^{2} / 2$ with boundary condition $m(\bar{p})=0$ and negative symmetry $m(p)=-m(-p)$, with closed form solution:

$$
m(p)=-\frac{p}{\lambda}+\frac{\bar{p}}{\lambda}\left(\frac{e^{\sqrt{2 \phi} \frac{p}{\bar{p}}}-e^{-\sqrt{2 \phi} \overline{\bar{p}}}}{e^{\sqrt{2 \phi}}-e^{-\sqrt{2 \phi}}}\right) \text { for all } p \in[-\bar{p}, \bar{p}] \quad \text { where } \quad \phi \equiv \lambda \bar{p}^{2} / \sigma^{2}
$$

The final element to define $\mathcal{M}(\delta)$ is the density of the invariant distribution $g(p)$ for a vector of price gaps $p \in \mathbb{R}^{n}$ which is directly implied by the invariant density of the squared price gaps $f(y)$, given in equation (6), and by the observation that in steady state the distribution of price gaps with $\|p\|^{2}=y$ is uniform on the $n$ dimensional hypersphere of

\footnotetext{
${ }^{31}$ This proposition can be extended in a straightforward way to this paper using the logic of Lemma 1.
} 
square radius $y$, whose closed form expression is given by:

$$
g\left(p_{1}, \ldots, p_{n}\right)=f\left(p_{1}^{2}+\cdots+p_{n}^{2}\right) \frac{\Gamma(n / 2)}{\pi^{n / 2}\left(p_{1}^{2}+\cdots+p_{n}^{2}\right)^{(n-2) / 2}} .
$$

Thus we can write

$$
\mathcal{M}(\delta)=\frac{1}{\epsilon n} \int \ldots \int m\left(p_{1}-\delta, \ldots, p_{n}-\delta\right) g\left(p_{1}, \ldots, p_{n}\right) d p_{1} \ldots d p_{n}
$$

Note that $\mathcal{M}$ takes $g(p)$ firms with price gap vector $p$ in steady state, shifts them down by $\delta$, which amounts to increasing the marginal nominal cost on impact, and then computes their contribution $m\left(p_{1}-\delta, \ldots, p_{n}-\delta\right)$.

Recall that, for any $n \geq 1$ we can index the steady state of an economy by three numbers, $N\left(\Delta p_{i}\right), \operatorname{Std}\left(\Delta p_{i}\right)$, and $\ell$, for which we can always find the values of $\left(\lambda, \psi, \sigma^{2}\right)$ to rationalize them. The next proposition shows that $\mathcal{M}$ can be normalized, i.e. written in terms of the values of an economy with one price change per year, and where the monetary shock is measured in terms of steady-state size of price changes.

Proposition 6 Consider an economy whose firms produce $n>1$ products, with steady-state statistics $\left(N\left(\Delta p_{i}\right), \operatorname{Std}\left(\Delta p_{i}\right), \ell\right)$. Then

$$
\mathcal{M}\left(\delta ; N\left(\Delta p_{i}\right), \operatorname{Std}\left(\Delta p_{i}\right), n, \ell\right)=\frac{S t d\left(\Delta p_{i}\right)}{N\left(\Delta p_{i}\right)} \mathcal{M}\left(\frac{\delta}{\operatorname{Std}\left(\Delta p_{i}\right)} ; 1,1, n, \ell\right)
$$

Equation (20) shows that keeping $(n, \ell)$ fixed, $\mathcal{M}$ can be scaled by the steady state frequency of price changes $N\left(\Delta p_{i}\right)$, and that the size of the monetary shock should be measured relative to steady state size of price changes $\operatorname{Std}\left(\Delta p_{i}\right)$.

\subsection{The case of a small monetary shock}

To focus on a small shock $\delta$, a realistic standard in this literature, we take the first order approximation to equation (15), using equation (20) we obtain $\mathcal{M}\left(\delta ; N\left(\Delta p_{i}\right), \operatorname{Std}\left(\Delta p_{i}\right), n, \ell\right) \approx$ $\delta / N\left(\Delta p_{i}\right) \mathcal{M}^{\prime}(0 ; 1,1, n, \ell)$. Thus for a small monetary shock, $\operatorname{Std}\left(\Delta p_{i}\right)$ has no effect on the cumulative output effect. The usefulness of the approach developed in this section is easily seen in the $n=1$ case. Using the closed form solution for $g(p)$ in equation (2) and the expression for $m(p)$ in equation (17) we can now analytically compute the cumulative effect of a small monetary shock of size $\delta$ using equation (19) and the approximation 
$\mathcal{M}(\delta) \approx \delta / N\left(\Delta p_{i}\right) \mathcal{M}^{\prime}(0)$ which yields

$$
\delta \mathcal{M}^{\prime}(0)=\frac{\delta}{\epsilon N\left(\Delta p_{i}\right)} \frac{e^{\sqrt{2 \phi}}+e^{-\sqrt{2 \phi}}}{\left(e^{\sqrt{2 \phi}}+e^{-\sqrt{2 \phi}}-2\right)^{2}}\left(e^{\sqrt{2 \phi}}+e^{-\sqrt{2 \phi}}-2-2 \phi\right) .
$$

This formula can be used to compare a Calvo model where $\ell \rightarrow 1$ (or $\phi \rightarrow \infty$, see equation (5)) with a Golosov-Lucas model, where $\ell \rightarrow 0$ (or $\phi \rightarrow 0$ ). Simple analysis shows that in Calvo we have $\delta \mathcal{M}^{\prime}(0)=\frac{\delta}{\epsilon N\left(\Delta p_{i}\right)}$, whereas in Golosov-Lucas we have $\delta \mathcal{M}^{\prime}(0)=\frac{\delta}{6 \epsilon N\left(\Delta p_{i}\right)}$, so that the cumulative output effect in these models differs by a factor of 6 . Interestingly, the number 6 is exactly the ratio between the kurtosis of price changes in each of these models. The next result shows that this result is general and applies to any $n \geq 1$ and $\ell \in(0,1)$.

Proposition 7 Consider an economy whose firms produce $n>1$ products, with steady-state statistics $N\left(\Delta p_{i}\right), \operatorname{Std}\left(\Delta p_{i}\right), \ell$ and a steady-state kurtosis of price changes $\operatorname{Kur}\left(\Delta p_{i}\right)$. For a small monetary shock $\delta$ we have the following first order Taylor expansion:

$$
\mathcal{M}\left(\delta ; N\left(\Delta p_{i}\right), S t d\left(\Delta p_{i}\right), n, \ell\right) \approx \frac{\delta}{\epsilon} \frac{K u r\left(\Delta p_{i}\right)}{6 N\left(\Delta p_{i}\right)}=\frac{\delta}{\epsilon} \frac{\sum_{i=2}^{\infty} \frac{1}{i ! \Gamma\left(\frac{n}{2}+i\right)}\left(\frac{\lambda \bar{y}}{2 \sigma^{2}}\right)^{i}}{\lambda \sum_{i=1}^{\infty} \frac{1}{i ! \Gamma\left(\frac{n}{2}+i\right)}\left(\frac{\lambda \bar{y}}{2 \sigma^{2}}\right)^{i}}
$$

This proposition is quite useful to explain what produces the different results that are found in the literature on the real effects of monetary policy. The proposition illustrates how it is possible for two models sharing similar features, e.g. calibrated to the same observables $N\left(\Delta p_{i}\right), \operatorname{Std}\left(\Delta p_{i}\right)$ and sharing the same preference specification (labor elasticity $1 / \epsilon$ ), to produce different predictions about the output effect: what is needed is that the model predicts a different kurtosis of price changes.

Recall from Proposition 4 that the shape of the size distribution of price changes, and hence kurtosis, depends only on $n$ and $\ell$. For a fixed $n$, kurtosis is increasing in $\ell$. Indeed, as $\ell$ goes to 1 then kurtosis goes to 6 , and hence we obtain $\mathcal{M}(\delta) \cong \delta /\left(\epsilon N\left(\Delta p_{i}\right)\right)$, which is the result produced by the Calvo pricing model. On the other extreme, as $\ell=0$ kurtosis equals $3 n /(n+2)$. This implies that, for instance, in the Golosov and Lucas case of $n=1$, the impact of monetary policy is $1 / 6$ of Calvo. Also, keeping $\ell=0$ and varying $n$ from 1 to $\infty$, the effect goes from $1 / 6$ to $1 / 2$ of Calvo. Note that in the case of $\ell=0$ and $n=\infty$ the model becomes Taylor's staggered price model or, equivalently, Reis (2006) model. Thus the purely multi product Taylor-Reis case $(\ell=0, n=\infty)$ delivers only half of the real effects compared to a purely Calvo model $(\ell=1)$, as further discussed below.

The discussion above makes clear that the assumption of non-gaussian shocks in Midrigan (2011) is quite crucial to obtain real effects that are closer to Calvo than to Golosov-Lucas. 
What is needed for the effects to be large is a large kurtosis, which Midrigan obtains by assuming a process for the shocks hitting the firm's costs that are fat-tailed. It can indeed be shown that introducing fat-tails in our version of the Golosov-Lucas model, through shocks to the marginal cost occurring with a Poisson intensity, leads to a formally similar problem to that of the model with free adjustment opportunities, as we do in Appendix H.

Figure 5: Cumulative output effect relative to Calvo pricing: $\operatorname{Kur}\left(\Delta p_{i}\right) / 6$

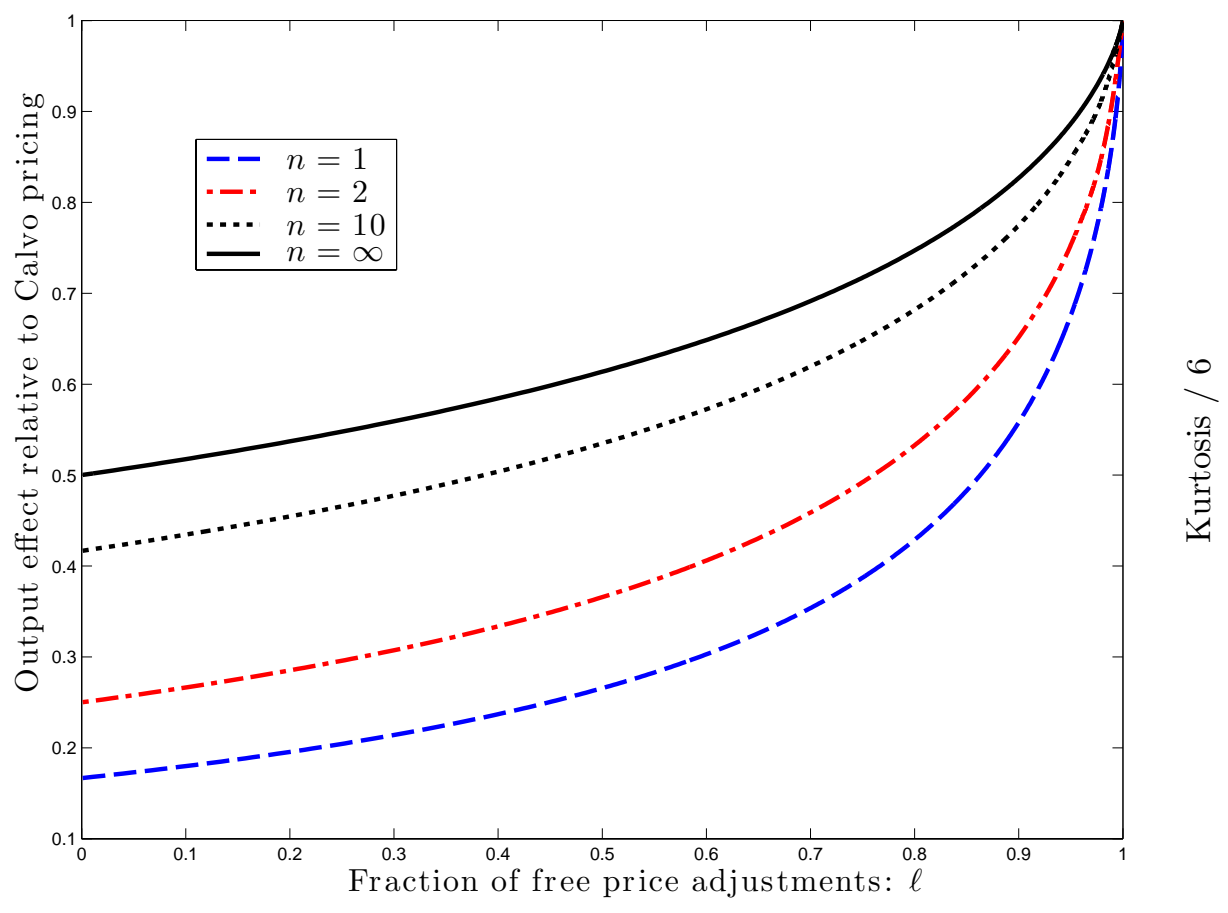

Figure 5 offers a richer systematic comparison of the real effects of monetary shocks as $n$ and $\ell$ vary: the vertical axis plots the real output effect produced by a small monetary shock relative to the effect produced by a Calvo model where $\ell=1$. Four curves are plotted in the figure, corresponding to $n=1,2,10, \infty$. It appears that the model behavior for $n=2$ remains quite close to the case where $n=1$, as was also seen from the analysis of the distribution of price changes. Instead, the model behavior for $n=10$ is quite close to that of a model where $n=\infty$. This is useful because the latter is quite tractable analytically, as discussed below. Figure 5 shows that at any level of $\ell$ the real output effect are smallest for $n=1$. As explained in Alvarez and Lippi (2014) a larger number of goods dampens the selection effect of monetary policy increasing the real output consequences of a monetary shock. Indeed at any level of $\ell$ the effect is increasing in $n$. The figure shows that fixing $n$ the output effect is increasing in $\ell$. In the limit, as $\ell \rightarrow 1$ the economy converges to a Calvo model where the real effects are largest and independent of $n$. 
It is interesting to notice that the curves plotted in the figure are convex. In particular, some analysis reveals that the slope of the curve as $\ell \rightarrow 1$ diverges to $+\infty$ for any level of $n$ (see the formula for $\mathcal{M}$ in the special case when $n=\infty$ in Appendix $\mathrm{P}$ ). The economic implication of this property is that a small deviation from Calvo pricing, i.e. a fraction of adjustment $\ell$ that is slightly below 1 is going to give rise to a large deviation from the real effects predicted by the Calvo pricing. That the relatively large real effects in Calvo are very sensitive to the introduction of a small amount of selection by firms regarding the timing of price changes, is also apparent in the CalvoPlus model of Nakamura and Steinsson (2010) (see their figure VII). Hence the finding seems robust as these models, and measure of real effects, are similar but not identical.

Additionally, equation (22), together with equation (3) and equation (5), completes the closed form solutions for $N\left(\Delta p_{i}\right), \operatorname{Std}\left(\Delta p_{i}\right), \operatorname{Kur}\left(\Delta p_{i}\right)$ and $\mathcal{M}^{\prime}(0)$ given parameters $n, \lambda, \sigma^{2}$ and threshold $\bar{y}$, which instead can be solved explicitly using Lemma 1 and Proposition 2.

We finish this section discussing the economic intuition of why there is a systematic relationship between kurtosis and the cumulative output effect of an monetary shock. As it is remarked in the literature, at least since the analysis in Golosov and Lucas (2007), the small output effect of a monetary shock in menu cost models is related to the degree to which the firms that change prices right after the shock are of a "selected" type. In particular, in Golosov and Lucas's model those firms are more likely to increase prices, and hence the aggregate price level adjusts fast initially. Instead, in Calvo's model the average price change of firms at any horizon $t$ of the impulse response is always the same, equal to $\delta$. We refer to this effect as "selection on size of price changes". This is captured in the steady state kurtosis of the distribution of price changes, since it is the proximity to the adjustment barriers that produces price changes that are platykurtic in menu-cost models. Yet this cannot be the full story, since all the models for which $n=\infty$ have that the average price changes of the firms at any horizon in the impulse response is equal to $\delta$. Nevertheless, the model with $\ell=0$ (Taylor's) produces half the cumulative output than the one with $\ell=1$ (Calvo). The difference across these models is in the timing of when firms adjust: in Taylor the distribution of times until adjustment is uniform, but in Calvo it is exponential, with a thicker right tail of firms that adjust very late. We refer to this effect as to the "selection in the times of price changes". This effect is also clearly captured by the steady state kurtosis, since a wider distribution of times to adjust produces a distribution of price changes that is a mixture of normals with different variances. Indeed our result in Proposition 7 extends to a more general class of models where selection concerns exclusively the times of adjustment (not the size), as in Carvalho and Schwartzman (2012) and Alvarez, Lippi, and Paciello (2012). 
Lack of sensitivity to inflation. While the model we have written is based on an economy which has a zero steady-state inflation, we argue that the characterization of kurtosis as well as the key result on the effect of monetary policy in Proposition 7 apply also for low rates of inflation. Indeed steady-state inflation has only second order effect on both the kurtosis of the price changes as well as the area under the IRF of output for a small monetary shock. In particular, we show that both the left and right hand side of equation (22) have a zero derivative with respect the steady-state inflation, evaluated at zero inflation. For this we consider an economy where the money supply grows at the rate $\mu$ and the steady state inflation rate and the growth rate of nominal wages both equal $\mu$. In this case the price gaps will evolve as $\mathrm{d} p_{i}(t)=-\mu \mathrm{d} t+\sigma \mathrm{d} W_{i}(t)$ where the negative sign of the drift reflects that wages grow at a constant rate. For this model we let $\operatorname{Kur}\left(\Delta p_{i} ; \mu\right), N\left(\Delta p_{i} ; \mu\right)$ be the kurtosis and frequency of price changes at steady state as a function of inflation $\mu$. Likewise, we consider a once and for all (unanticipated) increase in the level of money supply of size $\delta$, so that the path continues to grow at rate $\mu$ right after. We let $\mathcal{M}(\delta ; \mu)$ be the area-under the IRF of output after such monetary shock $\delta$, also indexed by the steady state inflation. We have the following result:

Proposition 8 Let $\mu$ be the steady state inflation rate. Then:

$$
0=\left.\frac{\partial \operatorname{Kur}\left(\Delta p_{i} ; \mu\right)}{\partial \mu}\right|_{\mu=0}=\left.\frac{\partial N\left(\Delta p_{i} ; \mu\right)}{\partial \mu}\right|_{\mu=0}=\left.\frac{\partial \mathcal{M}^{\prime}(0 ; \mu)}{\partial \mu}\right|_{\mu=0}
$$

and hence the derivative of both sides of equation (22) with respect to $\mu$ evaluated at $\mu=0$ is zero.

Hence, even though we have developed the result for zero inflation, this proposition shows that the results provide a good benchmark for a low inflation economy. We think this is important since developed economies have low but positive inflation rates. The idea behind the proof is to exploit the symmetry of both kurtosis $\operatorname{Kur}\left(\Delta p_{i} ; \mu\right)$ and the cumulative IRF $\mathcal{M}^{\prime}(0 ; \mu) \delta$ with respect to inflation $\mu$ around $\mu=0$.

To summarize, we showed that adding a small inflation has a negligible effect on the findings of the paper, including the main result in equation (1). This theoretical prediction is consistent with evidence on the small elasticity of several price setting statistics (such as the frequency, variance and kurtosis) provided in Gagnon (2009) and Alvarez et al. (2011).

\subsection{The case of a large monetary shock}

The dependence of the output effect on the size of the monetary shock is a hallmark of a menu cost models. The next proposition characterizes the smallest value of the monetary 
shock $\underline{\delta}$ for which all firms adjust prices immediately, in which case the impact response of the aggregate price level equal the monetary shock, $\mathcal{P}(\delta, 0)=\delta$ so that prices are flexible and there is no effect on output, $\mathcal{M}(\delta)=0$. The value of this threshold for the monetary shock is proportional to the $\operatorname{Std}\left(\Delta p_{i}\right)$ which properly scales the "size" of the monetary shocks, and to a constant of proportionality that is increasing in $\ell$.

Proposition 9 Define $\underline{\delta}$ as the smallest once-and-for-all monetary shock for which there is full price flexibility, i.e. $\mathcal{M}(\delta)=0$ for any $\delta \geq \underline{\delta}$. We have

$$
\underline{\delta}=2 \sqrt{\frac{\bar{y}}{n}}=2 \operatorname{Std}\left(\Delta p_{i}\right) \sqrt{\frac{\mathcal{L}^{-1}(\ell ; n)}{\ell}},
$$

where $\mathcal{L}(\cdot ; n)$ is given by equation (5). Fixing any $n \geq 1$, the ratio $\underline{\delta} /\left(2 \operatorname{Std}\left(\Delta p_{i}\right)\right)$ is a strictly increasing function of $\ell$, ranging from 1 to $\infty$ as $\ell$ varies from 0 to 1 .

Note that for $\ell=0$, we have $\underline{\delta}=2 \operatorname{Std}\left(\Delta p_{i}\right)$ for all $n \geq 1$, so the minimum shock is simply twice the standard deviation of prices. In general this threshold is increasing in $\ell$, becoming unbounded as the model gets closer to Calvo, i.e. $\lim _{\ell \rightarrow 1} \underline{\delta}=+\infty$ for all $n \geq 1$. This is intuitive since as $\ell$ increases more of the price changes are due to the (exogenous) free opportunity to adjust. Computing the value of $\underline{\delta}$ is immediate given the closed form expressions in Proposition 3 and Proposition 9. The analysis shows that: (i) for a given $\ell$ the threshold $\underline{\delta}$ is decreasing in $n$, and (ii) for all $n \geq 1$, even for $\ell=0.85$ the values for $\underline{\delta}$ are smaller than twice the corresponding value for $\ell=0$. In other words, $\underline{\delta}$ is a steep convex function of $\ell$, with an infinite positive slope as $\ell \rightarrow 1$. This finding implies that workings of a Calvo model are substantially different from the workings of a model that is close, but slightly below, the Calvo specification with $\ell=1$ (see Appendix $\mathrm{G}$ for a rigorous definition and analysis of the impact effect on the aggregate price level; Appendix $\mathrm{J}$ for more documentation and a proof of the proposition).

\section{Robustness and scope of results}

Our main results, namely equation (1), applies more generally. First, while the paper focused on an economy with ex-ante identical firms, we show in Appendix $\mathrm{R}$ that a straightforward modification of equation (1) holds in a model with ex ante heterogenous sectors that differ in the frequency of adjustment and/or the kurtosis of price changes. Second, equation (1) holds also in models with different price setting frictions. For instance, a small variation of our model assumes that the cost of a price adjustment upon a random opportunity is not free, but is just cheaper than the regular menu cost (see Appendix S). This setup is closer to the 
original CalvoPlus model by Nakamura and Steinsson (2010) than the one in the main text. Interestingly we found that equation (1) holds in that model too. Another variation considers a different class of models, within a "rational inattentiveness" setup where individual firms can observe their state only after paying a fixed "observation" cost. In Alvarez, Lippi, and Paciello (2011) we consider the case where firms face both observation and menu costs so that both Reis (2006) and Golosov and Lucas (2007) are special cases. It turns out that equation (1) holds in this model. In Alvarez, Lippi, and Paciello (2012) we consider the case where firms face random observation cost and no menu costs. This setup produces random adjustment times at the firm level, whose consequences for the propagation of monetary shocks have been studied by Mankiw and Reis (2002) and Carvalho and Schwartzman (2012). The last class of models does not feature any selection in the size of price changes but only in the times of adjustment. Moreover, while kurtosis is between 1 and 6 in our menu cost model, it is unbounded in these models. Interestingly, one can show that equation (1) holds in these class of models too.

Finally, we explored a setting where the cost shocks that trigger the price changes are not continuous, but occur infrequently and are "large". We refer to this setting as fat-tailed shocks, a setup that is close to the model of Gertler and Leahy (2008) and the version of Midrigan (2011) with non-gaussian shocks (see Appendix H for details). This setting introduces a wedge between the timing of price changes and the size-distribution of price changes that impairs equation (1): kurtosis is not anymore a sufficient statistic about the price setting behavior, because it also reflects the assumptions about the shape of the large (infrequent) shocks. Compared to our model, these models require more observations to be identified, such as direct information on the shape of the cost shocks faced by firms. We leave this exploration for future work. Yet another extension which we leave for the future consists in solving the model under the assumption that the cost shocks that hit the goods sold by the firm are partially correlated.

\section{References}

Alvarez, Fernando, Martin Gonzalez-Rozada, Andres Neumeyer, and Martin Beraja. 2011. "From Hyperinflation to Stable Prices: Argentinas evidence on menu cost models." manuscript, University of Chicago.

Alvarez, Fernando E. and Francesco Lippi. 2013. "The demand of liquid assets with uncertain lumpy expenditures." Journal of Monetary Economics 60 (7):753-770.

$82(1): 89-135$. 
Alvarez, Fernando E., Francesco Lippi, and Luigi Paciello. 2011. "Optimal price setting with observation and menu costs." The Quarterly Journal of Economics 126 (4):1909-1960.

—. 2012. "Monetary Shocks in a Model with Inattentive Producers." Eief working papers series, Einaudi Institute for Economics and Finance (EIEF).

Alvarez, Fernando E., Francesco Lippi, and Andrea Pozzi. 2014. "A note on the sizedistribution of price changes in a large US dataset." Available on Lippi's website, EIEF.

Alvarez, Luis J., Emmanuel Dhyne, Marco Hoeberichts, Claudia Kwapil, Herve Le Bihan, Patrick Lunnemann, Fernando Martins, Roberto Sabbatini, Harald Stahl, Philip Vermeulen, and Jouko Vilmunen. 2006. "Sticky Prices in the Euro Area: A Summary of New Micro-Evidence." Journal of the European Economic Association 4 (2-3):575-584.

Arkolakis, Costas, Arnaud Costinot, and Andres Rodriguez-Clare. 2012. "New Trade Models, Same Old Gains?" American Economic Review 102 (1):94-130.

Barro, Robert J. 1972. "A Theory of Monopolistic Price Adjustment." Review of Economic Studies 39 (1):17-26.

Baudry, L., H. Le Bihan, P. Sevestre, and S. Tarrieu. 2007. "What do Thirteen Million Price Records have to Say about Consumer Price Rigidity?" Oxford Bulletin of Economics and Statistics 69 (2):139-183.

Berardi, Nicoletta, Erwan Gautier, and Hervé Le Bihan. 2013. "More facts about prices: France before and during the great recession." Working Paper 425, Banque de France.

Bhattarai, Saroj and Raphael Schoenle. 2014. "Multiproduct Firms and Price-Setting: Theory and Evidence from U.S. Producer Prices." Journal of Monetary Economics forthcoming.

Bronnenberg, Bart J., Michael W. Kruger, and Carl F. Mela. 2008. "The IRI marketing data set." Marketing Science 27 (4):745-748.

Caballero, Ricardo J. and Eduardo M.R.A. Engel. 2007. "Price stickiness in Ss models: New interpretations of old results." Journal of Monetary Economics 54 (Supplement):100-121.

Calvo, Guillermo A. 1983. "Staggered prices in a utility-maximizing framework." Journal of Monetary Economics 12 (3):383-398.

Carvalho, Carlos. 2006. "Heterogeneity in Price Stickiness and the Real Effects of Monetary Shocks." The B.E. Journal of Macroeconomics 6 (3):1-58.

Carvalho, Carlos and Felipe Schwartzman. 2012. "Selection and Monetary Non-Neutrality in Time-Dependent Pricing Models." Tech. rep., FRB of Richmond.

Cavallo, Alberto. 2010. "Scraped Data and Sticky Prices." Tech. rep., MIT Sloan. 
Chakrabarti, Rajesh and Barry Scholnick. 2007. "The mechanics of price adjustment: new evidence on the (un) importance of menu costs." Managerial and Decision Economics 28 (7):657-668.

Chari, V. V., Patrick J. Kehoe, and Ellen R. McGrattan. 2000. "Sticky Price Models of the Business Cycle: Can the Contract Multiplier Solve the Persistence Problem?" Econometrica 68 (5):1151-1180.

Chen, Haipeng, Daniel Levy, Sourav Ray, and Mark Bergen. 2008. "Asymmetric price adjustment in the small." Journal of Monetary Economics 55 (4):728-737.

Chetty, Raj. 2009. "Sufficient Statistics for Welfare Analysis: A Bridge Between Structural and Reduced-Form Methods." Annual Review of Economics 1 (1):451-488.

Christopoulou, Rebekka and Philip Vermeulen. 2012. "Markups in the Euro area and the US over the period 19812004: a comparison of 50 sectors." Empirical Economics 42 (1):53-77.

Dixit, Avinash. 1991. "Analytical Approximations in Models of Hysteresis." Review of Economic Studies 58 (1):141-51.

Dotsey, Michael, Robert G. King, and Alexander L. Wolman. 1999. "State-Dependent Pricing And The General Equilibrium Dynamics Of Money And Output." The Quarterly Journal of Economics 114 (2):655-690.

2009. "Inflation and Real Activity with firm-level productivity shocks." Tech. rep., Federal Reserve Bank of Richmond.

Dutta, Shantanu, Mark Bergen, Daniel Levy, and Robert Venable. 1999. "Menu Costs, posted prices and multiproduct retailers." The Journal of Money, Credit and Banking 31 (4):683-703.

Eichenbaum, Martin, Nir Jaimovich, and Sergio Rebelo. 2011. "Reference Prices, Costs, and Nominal Rigidities." American Economic Review 101 (1):234-62.

Eichenbaum, Martin S., Nir Jaimovich, Sergio Rebelo, and Josephine Smith. 2014. "How Frequent Are Small Price Changes?" American Economic Journal: Macroeconomics $6(2): 137-155$.

Gagnon, Etienne. 2009. "Price Setting During Low and High Inflation: Evidence from Mexico." Quarterly Journal of Economics 124 (3):1221-1263.

Gertler, Mark and John Leahy. 2008. "A Phillips Curve with an Ss Foundation." Journal of Political Economy 116 (3):533-572.

Golosov, Mikhail and Robert E. Jr. Lucas. 2007. "Menu Costs and Phillips Curves." Journal of Political Economy 115:171-199.

Kashyap, Anil K. 1995. "Sticky Prices: New Evidence from Retail Catalogs." The Quarterly Journal of Economics 110 (1):245-74. 
Kehoe, Patrick J. and Virgiliu Midrigan. 2007. "Sales and the real effects of monetary policy." Working Papers 652, Federal Reserve Bank of Minneapolis.

Kiley, Michael. 2002. "Partial Adjustment and Staggered Price Setting." Journal of Money, Credit and Banking 34 (2):283-98.

Klenow, J. Peter and Benjamin Malin. 2010. "Microeconomic Evidence on Price-Setting." Handbook of monetary economics 3:231-284.

Klenow, Peter J. and Oleksiy Kryvtsov. 2008. "State-Dependent or Time-Dependent Pricing: Does It Matter for Recent U.S. Inflation?" The Quarterly Journal of Economics $123(3): 863-904$.

Levy, Daniel, Mark Bergen, Shantanu Dutta, and Robert Venable. 1997. "The Magnitude of Menu Costs: Direct Evidence from Large U.S. Supermarket Chains." The Quarterly Journal of Economics 112 (3):791-825.

Mankiw, N. Gregory and Ricardo Reis. 2002. "Sticky Information Versus Sticky Prices: A Proposal To Replace The New Keynesian Phillips Curve." The Quarterly Journal of Economics 117 (4):1295-1328.

Midrigan, Virgiliu. 2009. "Menu Costs, Multi-Product Firms, and Aggregate Fluctuations." Working paper, NYU.

—. 2011. "Menu Costs, Multi-Product Firms, and Aggregate Fluctuations." Econometrica, 79 (4):1139-1180.

Nakamura, Emi and Jon Steinsson. 2008. "Five Facts about Prices: A Reevaluation of Menu Cost Models." The Quarterly Journal of Economics 123 (4):1415-1464.

. 2010. "Monetary Non-Neutrality in a Multisector Menu Cost Model." The Quarterly Journal of Economics 125 (3):961-1013.

Reis, Ricardo. 2006. "Inattentive producers." Review of Economic Studies 73 (3):793-821.

Taylor, John B. 1980. "Aggregate Dynamics and Staggered Contracts." Journal of Political Economy 88 (1):1-23.

Vavra, Joseph. 2013. "Inflation Dynamics and Time-Varying Volatility: New Evidence and an Ss Interpretation." The Quarterly Journal of Economics (forthcoming).

Wulfsberg, F. 2010. "Price adjustments and inflation-evidence from Norwegian consumer price data 1975-2004." Working paper, Bank of Norway.

Zaitsev, V.F. and A.D. Polyanin. 2003. Handbook of Exact Solutions for Ordinary Differential Equations. Chapman and Hall, CRC Press.

Zbaracki, Mark J., Mark Ritson, Daniel Levy, Shantanu Dutta, and Mark Bergen. 2004. "Managerial and Customer Costs of Price Adjustment: Direct Evidence from Industrial Markets." The Review of Economics and Statistics 86 (2):514-533. 


\section{A Proofs}

Proof. (of Proposition 1). Let $p(0)=0$. Define $x(t) \equiv\|p(t)\|^{2}-n \sigma^{2} t$ for $t \geq 0$. Using Ito's lemma we can verify that the drift of $\|p\|^{2}$ is $n \sigma^{2}$, and hence $x(t)$ is a Martingale. By the optional sampling theorem $x(\tau)$, the process stopped at $\tau$, is also a martingale. Then $\mathbb{E}[x(\tau) \mid p(0)]=\mathbb{E}\left[\|p(\tau)\|^{2} \mid p(0)\right]-n \sigma^{2} \mathbb{E}[\tau \mid p(0)]=x(0)=0$ and since $N\left(\Delta p_{i}\right)=$ $1 / \mathbb{E}[\tau \mid p(0)]$ and $\operatorname{Var}\left(\Delta p_{i}\right)=\mathbb{E}\left[\|p(\tau)\|^{2} \mid p(0)\right] / n$ we obtain the desired result.

Proof. (of Lemma 1). First, note that since two value functions differ by a constant, then all their derivatives are identical. Hence, if the one for the discount rate and arrival rate of free adjustment $(r+\lambda, 0)$ satisfies value matching and smooth pasting, so does the one for discount rate and arrival rate of free adjustment $(r, \lambda, 0)$, for the same boundary. Second, consider the range of inaction, subtracting the value function for the problem with parameters $(r+\lambda, 0)$ from the one with parameters $(r, \lambda)$, and using that all the derivatives are identical, one verifies that if the Bellman equation holds for the problem with $(r+\lambda, 0)$, so it does for the problem with $(r, \lambda)$.

Proof. (of Proposition 2 ). The first part is straightforward given Lemma 1 and Proposition 3 in Alvarez and Lippi (2014). The second part is derived from the following implicit expression determining $\bar{y}$ (see the proof of Proposition 3 in Alvarez Lippi for the derivation):

$$
\psi=\frac{B}{r+\lambda} \bar{y}\left[1-\frac{\frac{2 \sigma^{2}(n+2)}{r+\lambda} \bar{y}+\bar{y}^{2}+\bar{y}^{2} \sum_{i=1}^{\infty} \kappa_{i}(r+\lambda)^{i} \bar{y}^{i}}{\frac{2 \sigma^{2}(n+2)}{r+\lambda} \bar{y}+2 \bar{y}^{2}+\bar{y}^{2} \sum_{i=1}^{\infty} \kappa_{i}(i+2)(r+\lambda)^{i} \bar{y}^{i}}\right]
$$

where $\kappa_{i}=(r+\lambda)^{-i} \prod_{s=1}^{i} \frac{1}{\sigma^{2}(s+2)(n+2 s+2)}$. So we can write this expression as: $\psi=\frac{B}{r+\lambda} \bar{y}$ $\left[1-\xi\left(\sigma^{2}, r+\lambda, n, \bar{y}\right)\right]$ where $\xi\left(\sigma^{2}, r+\lambda, n, \bar{y}\right)$ is given by:

$$
\xi\left(\sigma^{2}, r+\lambda, n, \bar{y}\right) \equiv \frac{\frac{2 \sigma^{2}(n+2)}{r+\lambda} \bar{y}+\bar{y}^{2}+\bar{y}^{2} \sum_{i=1}^{\infty} \kappa_{i}(r+\lambda)^{i} \bar{y}^{i}}{\frac{2 \sigma^{2}(n+2)}{r+\lambda} \bar{y}+2 \bar{y}^{2}+\bar{y}^{2} \sum_{i=1}^{\infty} \kappa_{i}(i+2)(r+\lambda)^{i} \bar{y}^{i}}
$$

Since $\bar{y} \rightarrow \infty$ as $\psi \rightarrow \infty$ then we can define the limit:

$$
\lim _{\psi \rightarrow \infty} \frac{\psi}{\bar{y}}=\frac{B}{r+\lambda}\left[1-\lim _{\bar{y} \rightarrow \infty} \xi\left(\sigma^{2}, r+\lambda, n, \bar{y}\right)\right]
$$

Simple analysis can be used to show that $\lim _{\bar{y} \rightarrow \infty} \xi\left(\sigma^{2}, r+\lambda, n, \bar{y}\right)=0$ which gives the expression in the proposition (see the Online Appendix E for a detailed derivation).

Proof. (of Proposition 3 ). To characterize $N\left(\Delta p_{i}\right)$ we write the Kolmogorov backward equation for the expected time between adjustments $\mathcal{T}(y)$ which solves: $\lambda \mathcal{T}(y)=$ $1+n \sigma^{2} \mathcal{T}^{\prime}(y)+2 y \sigma^{2} \mathcal{T}^{\prime \prime}(y)$ for $y \in(0, \bar{y})$ and $\mathcal{T}(\bar{y})=0$ (see Appendix $\mathrm{F}$ for a discussion of the solution to this equation). Then the expected number of adjustments is given by $N\left(\Delta p_{i}\right)=1 / \mathcal{T}(0)$, subject to $\mathcal{T}(0)<\infty$. 
The solution of the second order ODE for $\mathcal{T}(y)$ has a power series representation: $\mathcal{T}(y)=$ $\sum_{i=0}^{\infty} \alpha_{i} y^{i}$, for $y \in[0, \bar{y}]$, with the following conditions on its coefficients $\left\{\alpha_{i}\right\}: \alpha_{1}=$ $\frac{\lambda \alpha_{0}-1}{n \sigma^{2}}, \quad \alpha_{i+1}=\frac{\lambda}{(i+1) \sigma^{2}(n+2 i)} \alpha_{i}, \quad$ for $i \geq 1$ and where $0<\alpha_{0}<1 / \lambda$ is chosen so that $0 \geq \alpha_{i}$ for $i \geq 1, \lim _{i \rightarrow \infty} \frac{\alpha_{i+1}}{\alpha_{i}}=0$ and $0=\sum_{i=0}^{\infty} \alpha_{i} \bar{y}^{i}$. Moreover, $\mathcal{T}(0)=\alpha_{0}$ is an increasing function of $\bar{y}$ since $\alpha_{0}$ solves:

$$
0=\alpha_{0}+\frac{\left(\alpha_{0}-1 / \lambda\right)}{n}\left(\frac{\bar{y} \lambda}{\sigma^{2}}\right)\left[1+\sum_{i=1}^{\infty}\left(\prod_{k=1}^{i} \frac{1}{(k+1)(n+2 k)}\right)\left(\frac{\bar{y} \lambda}{\sigma^{2}}\right)^{i}\right]
$$

Note that for $i \geq 1: \alpha_{i}=\alpha_{i} /[i !(n / 2+i)]\left(\lambda /\left(2 \sigma^{2}\right)\right)$, and using the properties of the $\Gamma$ function

$$
\alpha_{i}=\Gamma(n / 2) /\left(\Gamma(n / 2+i)\left(\lambda /\left(2 \sigma^{2}\right)\right)^{i}\left(\alpha_{0}-1 / \lambda\right) .\right.
$$

Solving for $\alpha_{0}$ and using $\mathcal{L} \equiv \lambda / N\left(\Delta p_{i}\right)=\lambda \mathcal{T}(0)=\lambda \alpha_{0}$. Thus

$$
\ell=\left(\sum_{i=1}^{\infty} \frac{\Gamma\left(\frac{n}{2}\right)}{i ! \Gamma\left(\frac{n}{2}+i\right)}\left(\frac{\lambda \bar{y}}{2 \sigma^{2}}\right)^{i}\right) /\left(\sum_{i=0}^{\infty} \frac{\Gamma\left(\frac{n}{2}\right)}{i ! \Gamma\left(\frac{n}{2}+i\right)}\left(\frac{\lambda \bar{y}}{2 \sigma^{2}}\right)^{i}\right)
$$

which is equation (5).

Proof. (of Proposition 4). We first state a lemma about the density $f(y)$.

Lemma 2 Let $f\left(y ; n, \frac{\lambda}{\sigma^{2}}, \bar{y}\right)$ be the density of $y \in[0, \bar{y}]$ in equation (6) satisfying the boundary conditions. For any $k>0$ we have: $f\left(y ; n, \frac{\lambda}{\sigma^{2}}, \bar{y}\right)=\frac{1}{k} f\left(\frac{y}{k} ; n, \frac{\lambda k}{\sigma^{2}}, \frac{\bar{y}}{k}\right)$.

Proof. (of Lemma 2 ). Consider the function $f\left(y ; n, \frac{\lambda}{\sigma^{2}}, \bar{y}\right)$ solving equation (6) (and boundary conditions) for given $n, \frac{\lambda}{\sigma^{2}}, \bar{y}$. Without loss of generality set $\sigma^{\prime}=\sigma$ and consider $\bar{y}^{\prime}=\bar{y} / k$ and $\lambda^{\prime}=\lambda k$. Notice that by setting $C_{1}^{\prime}=C_{1} k$ and $C_{2}^{\prime}=C_{2} k$ we verify that the boundary conditions hold (because $C_{1}^{\prime} / C_{2}^{\prime}=C_{1} / C_{2}$ ) and that (6) holds (which is readily verified by a change of variable).

We now prove the proposition. Let $w\left(\Delta p_{i} ; n, \ell, S t d\left(\Delta p_{i}\right)\right)$ be the density function in equation (8). Next we verify equation (9). From the first term in equation (8) notice that

$$
(1-\ell) \omega\left(\Delta p_{i} ; \bar{y}\right)=s(1-\ell) \omega\left(s \Delta p_{i} ; s^{2} \bar{y}\right)
$$

where the first equality uses the homogeneity of degree -1 of $\omega\left(\Delta p_{i} ; y\right)$ (see equation $\left.(7)\right)$. From the second term in equation (8) for $n \geq 2$

$$
\ell \int_{0}^{\bar{y}} \omega\left(\Delta p_{i} ; y\right) f\left(y ; n, \frac{\lambda}{\sigma^{2}}, \bar{y}\right) d y=\ell \int_{0}^{\bar{y}} s \omega\left(s \Delta p_{i} ; s^{2} y\right) s^{2} f\left(y s^{2} ; n, \frac{\lambda}{s^{2} \sigma^{2}}, \bar{y} s^{2}\right) d y
$$

where the first equality follows from Lemma 2 for $k=1 / s^{2}$, and the homogeneity of degree -1 of $\omega(\cdot, \cdot)$. Further we note

$$
\ell \int_{0}^{\bar{y}} s \omega\left(s \Delta p_{i} ; s^{2} y\right) s^{2} f\left(y s^{2} ; n, \frac{\lambda}{s^{2} \sigma^{2}}, \bar{y} s^{2}\right) d y=s^{3} \ell \int_{0}^{\bar{y}} \omega\left(s \Delta p_{i} ; s^{2} y\right) f\left(y s^{2} ; n, \frac{\lambda^{\prime}}{\sigma^{\prime 2}}, \bar{y}^{\prime}\right) d y
$$


where $\frac{\lambda^{\prime} \bar{y}^{\prime}}{\sigma^{\prime 2}}=\frac{\lambda \bar{y}}{\sigma^{2}}$, so that $\ell$ is the same across the two economies. Using $z=y s^{2}$

$$
s^{3} \ell \int_{0}^{\bar{y}} \omega\left(s \Delta p_{i} ; s^{2} y\right) f\left(y s^{2} ; n, \frac{\lambda^{\prime}}{\sigma^{\prime 2}}, \bar{y}^{\prime}\right) d y=s \ell \int_{0}^{\bar{y}^{\prime}} \omega\left(s \Delta p_{i} ; z\right) f\left(z ; n, \frac{\lambda^{\prime}}{\sigma^{\prime 2}}, \bar{y}^{\prime}\right) d z
$$

where $\bar{y}^{\prime}=s^{2} \bar{y}$, which completes the verification of equation (9).

Proof. (of Proposition 5). To obtain the expression in equation (11) we use the characterization of $\ell=\mathcal{L}\left(\frac{\lambda \bar{y}}{n \sigma^{2}}, n\right)$ of Proposition 3, it is equivalent to fix a value of $\phi \equiv \frac{\lambda \bar{y}}{n \sigma^{2}}$. We let the optimal decision rule be $\bar{y}\left(\psi / B, \sigma^{2}, r+\lambda, n\right)$ so that we have:

$$
\bar{y}\left(\frac{\psi}{B}, \sigma^{2}, r+\lambda, n\right) \frac{\lambda}{n \sigma^{2}}=\phi
$$

To be consistent with $\operatorname{Var}\left(\Delta p_{i}\right)$ and $N\left(\Delta p_{i}\right)$ we have, using Proposition 1 and $\ell=\mathcal{L}(\phi, n)$ :

$$
N\left(\Delta p_{i}\right)=\lambda / \mathcal{L}(\phi, n) \text { and } \frac{\lambda}{\sigma^{2}}=\mathcal{L}(\phi, n) / \operatorname{Var}\left(\Delta p_{i}\right)
$$

Thus, after taking $r \downarrow 0$ and using the expression above we can write:

$$
\bar{y}\left(\frac{\psi}{B}, N\left(\Delta p_{i}\right) \operatorname{Var}\left(\Delta p_{i}\right), \ell N\left(\Delta p_{i}\right), n\right) \frac{\ell}{n \operatorname{Var}\left(\Delta p_{i}\right)}=\mathcal{L}^{-1}(\ell ; n)
$$

Fixing $n$ and $\ell$ and totally differentiating this expression with respect to $\left(\psi / B, N\left(\Delta p_{i}\right), \operatorname{Var}\left(\Delta p_{i}\right)\right)$, and denoting by $\eta_{\psi}, \eta_{\sigma^{2}}, \eta_{\lambda}$ the elasticities of $\bar{y}$ with respect to $\psi / B, \sigma^{2}, \lambda$ we have:

$$
\eta_{\psi} \hat{\psi}+\eta_{\sigma^{2}}\left(\hat{N}\left(\Delta p_{i}\right)+\hat{\operatorname{Var}}\left(\Delta p_{i}\right)\right)+\eta_{\lambda} \hat{N}\left(\Delta p_{i}\right)=\hat{\operatorname{Var}}\left(\Delta p_{i}\right)
$$

where a hat denotes a proportional change. Using Proposition 3-(iv) in Alvarez and Lippi (2014) and Lemma 1 we have that these elasticities are related by: $\eta_{\lambda}=2 \eta_{\psi}-1$ and $\eta_{\sigma^{2}}=$ $1-\eta_{\psi}$. Thus $\eta_{\psi} \hat{\psi}+\left(1-\eta_{\psi}\right)\left(\hat{N}\left(\Delta p_{i}\right)+\hat{\operatorname{V}}\right.$ ar $\left.\left(\Delta p_{i}\right)\right)+\left(2 \eta_{\psi}-1\right) \hat{N}\left(\Delta p_{i}\right)=\hat{\operatorname{V}} \operatorname{ar}\left(\Delta p_{i}\right)$. Rearranging and canceling terms: $\eta_{\psi} \hat{\psi}+\eta_{\psi} \hat{N}\left(\Delta p_{i}\right)-\eta_{\psi} \hat{V} \operatorname{ar}\left(\Delta p_{i}\right)=0$. Dividing by $\eta_{\psi}$ we obtain that $\hat{\psi}=\hat{\operatorname{Var}}\left(\Delta p_{i}\right)-\hat{N}\left(\Delta p_{i}\right)$. Additionally, since $\bar{y}$ is a function of $\psi / B$, then we can write $\psi / n=B\left(\operatorname{Var}\left(\Delta p_{i}\right) / N\left(\Delta p_{i}\right)\right) \Psi(n, \ell)$ for some function $\Psi(n, \ell)$.

That $\psi \rightarrow \infty$ as $\ell \rightarrow 1$ follows because $\mathcal{L}(\phi, n) \rightarrow 1$ as $\phi \rightarrow \infty$ and because, by Proposition 3 -(i) in Alvarez and Lippi (2014), $\bar{y}$ is increasing in $\psi$ and has range and domain $[0, \infty)$. For $\lambda=0$ and $N\left(\Delta p_{i}\right)>0$ we obtain: $\frac{\psi}{n}=B \frac{V(\Delta p)}{N\left(\Delta p_{i}\right)} \frac{n}{2(n+2)}$. This follows from using the square root approximation of $\bar{y}$ for small $\psi(\lambda+r)^{2}$, the expression for $N\left(\Delta p_{i}\right)=n \sigma^{2} / \bar{y}$ and Proposition 1, i.e. $N\left(\Delta p_{i}\right) \operatorname{Var}\left(\Delta p_{i}\right)=\sigma^{2}$. To obtain the expression for $\Psi(n, 0)$ we use Proposition 6 in Alvarez and Lippi (2014) where it is shown that for $\lambda=0$ then $\operatorname{Kur}\left(\Delta p_{i}\right)=$ $3 n /(n+2)$.

Proof. (of Proposition 6). For any $p \in \mathbb{R}^{n}$ with $\|p\|^{2} \leq \bar{y}$, we write $m(p ; \bar{y}, \sigma, \lambda)$ to emphasize the dependence on $(\bar{y}, \sigma, \lambda)$. A guess and verify strategy can be used to show the following 
scaling property of the function $m$ : Let $k>0$, then for all $p \in \mathbb{R}^{n}$ with $\|p\|^{2} \leq \bar{y}$ :

$$
m\left(k p ; k^{2} \bar{y}, k \sigma, \lambda\right)=k m(p ; \bar{y}, \sigma, \lambda) \quad \text { and } \quad m(p ; \bar{y}, \sigma \sqrt{k}, \lambda k)=\frac{1}{k} m(p ; \bar{y}, \sigma, \lambda) .
$$

It is straightforward to verify that this function satisfies the ODE and boundary conditions for $m(p)$ (see e.g. the one in the main text for the $n=1$ case). Recall the homogeneity of $f(y)$ stated in Lemma 2. Finally, note that the density $g(p)$ can be expressed as a function of the density $f(y)$ given in equation (6) and the density of the sum of $n$ coordinates of a random variable uniformly distributed on a $n$ dimensional hypersphere of square radius y, as obtained in Equation 21 in Alvarez and Lippi (2014). These properties applied to equation (19) establish the scaling property stated in the proposition.

Proof. (of Proposition 7). For the cases of $n=1$ and $n=\infty$, in Appendix P we give closed form expressions for the kurtosis $\operatorname{Kur}\left(\Delta p_{i}\right)$ for any $\ell \in(0,1)$ and for the cumulative output effect, $\mathcal{M}(\delta)$ from which it is immediate to verify that they are proportional to each other, as stated in the proposition.

The next lemma gives a simple expression for $\mathcal{M}^{\prime}(0)$, which holds for all $1 \leq n<\infty$ and $0 \leq \ell \leq 1$

Lemma 3 Let $\mathcal{M}(\cdot ; n)$ be the area under the IRF of output and $f(\cdot ; n)$ be the density of the invariant distribution for an economy with $n$ products and parameters $\left(\bar{y}, \lambda, \sigma^{2}\right)$. Let $\mathcal{T}_{n+2}(y)$ be the expected time until either $y(t)$ hits $\bar{y}$ or that until there is a free adjustment opportunity, whichever happens first, starting at $y(0)=y$, for an economy with $n+2$ products and the same parameters $\left(\bar{y}, \lambda, \sigma^{2}\right)$. Then

$$
\mathcal{M}^{\prime}(0 ; n)=\frac{1}{\epsilon} \int_{0}^{\bar{y}}\left[\mathcal{T}_{n+2}(y)+\frac{2}{n} \mathcal{T}_{n+2}^{\prime}(y) y\right] f(y ; n) d y
$$

The function $\mathcal{T}_{n}(y)$ is characterized in the proof of Proposition 3 where we give an explicit power series representation for this function. The proof of Lemma 3 can be found in Appendix K. It uses a characterization of $m(p)$ in terms of a two dimensional vector $(z, y)$, where $z$ is the sum of the $n$ coordinates of $p$. The function $m(z, y)$ solves a PDE whose solution can be expressed in terms of $\mathcal{T}_{n}(y)$.

For $1<n<\infty$ and $\ell=0$ we note that Lemma 3 simplifies substantially since $\mathcal{T}_{n+2}(y)=$ $\left(\bar{y}-y /\left(2 n \sigma^{2}\right), \operatorname{Kur}\left(\Delta p_{i}\right)=3 n /(2+n), N\left(\Delta p_{i}\right)\right)=n \sigma^{2} / \bar{y}$, and $f$ has a simple closed form expression (all expressions are given in Alvarez and Lippi (2014)). Using these expressions to explicitly solve for the right hand side of equation (25) it is immediate to verify that equation (22) holds, see Appendix Q for the details.

For $1<n<\infty$ and $\ell=1$, with $\lambda>0$ and $\sigma^{2}>0$, using Proposition 3 it must be the case that $\bar{y}=\infty$. In this case, $N\left(\Delta p_{i}\right)=\lambda \ell=\lambda$, and the distribution of price changes is independent across each of the $n$ products, and given by a Laplace distribution, which has kurtosis 6 . Likewise $\mathcal{T}_{n+2}(y)=1 / \lambda$ for all $y \geq 0$. Thus, using equation (25) we obtain the desired result.

Finally we consider the case of $1<n<\infty$ and $0<\ell<1$. Using the representation shown in Proposition 6 for $\mathcal{M}(\delta)$ and differentiating with respect to $\delta$, and evaluating at $\delta=0$, then $\mathcal{M}^{\prime}(0)$ depends only on two parameters: $n$ and $\ell$. Likewise, using the property of $f$ in 
Proposition 4, the kurtosis of price changes also depends only on the same two parameters: $n$ and $\ell$. Therefore, for a given $n>1$, the verification of Proposition 7 requires to check that $\operatorname{Kur}\left(\Delta p_{i}\right) /\left(6 N\left(\Delta p_{i}\right)\right)$ and $\mathcal{M}^{\prime}(0)$ are the same at all values of $\ell \in(0,1)$. Using Proposition 3 , it is equivalent to check the equality for any $1<n<\infty$ and $0<\phi<\infty$ where $\phi=\lambda \bar{y} /\left(n \sigma^{2}\right)$.

For $\operatorname{Kur}\left(\Delta p_{i}\right) /\left(N\left(\Delta p_{i}\right)\right)$, the left hand side of equation (22), we have a closed form solution in equation (34). This solution is derived in two steps. First we derive a expression for $\operatorname{Kur}\left(\Delta p_{i}\right)$. This is done by noticing that

$$
\operatorname{Kur}\left(\Delta p_{i}\right)=\frac{\mathbb{E}\left(\Delta p_{i}^{4}(\tau) \mid y(0)=0\right)}{\operatorname{Var}\left(\Delta p_{i}\right)^{2}}=\frac{Q(0)}{\frac{\sigma^{4}}{N\left(\Delta p_{i}\right)^{2}}}=\frac{\left(\lambda / \sigma^{2}\right)^{2} Q(0)}{(\mathcal{L}(\phi, n))^{2}}
$$

where $\tau$ is the stopping time associated with a price change, and where $Q(y)$ is the expected fourth moment at the time of adjustment $\tau$ conditional on having today a squared price gap $y$, i.e.

$$
Q(y)=\mathbb{E}\left(\Delta p_{i}^{4}(\tau) \mid y(0)=y\right)=\frac{3}{(n+2) n} \mathbb{E}\left(y^{2}(\tau) \mid y(0)=y\right)
$$

where $y(\tau)$ is the value of the squared price gap at the stopping time. Notice that for $y \in[0, \bar{y}]$ the function $Q(y)$ obeys the o.d.e.:

$$
\lambda Q(y)=\lambda \frac{3 y^{2}}{(n+2) n}+Q^{\prime}(y) n \sigma^{2}+Q^{\prime \prime}(y) 2 \sigma^{2} y
$$

with boundary condition $Q(\bar{y})=\frac{3 \bar{y}^{2}}{(n+2) n}$. The solution of $Q$ has a a power series representation which is easily obtained by matching coefficients and using the boundary conditions. The second step is to use that $\operatorname{Kur}\left(\Delta p_{i}\right) / N\left(\Delta p_{i}\right)=(1 / \lambda) \mathcal{L}(\phi, n) \operatorname{Kur}\left(\Delta p_{i}\right)$ and the solution for $\mathcal{L}$ in Proposition 3. This gives expression in the right had side of equation (22). See Appendix D for more details in the algebra.

For the right hand side of equation (25), we first characterize $\mathcal{T}_{n+2}(y)+\frac{2}{n} \mathcal{T}_{n+2}^{\prime}(y) y$ and $f(y ; n)$. In the proof of Proposition 3 we developed a power series representation of $\mathcal{T}_{n+2}(y)$. Using this power series it is immediate to obtain a power series representation of $\mathcal{T}_{n+2}(y)+$ $\frac{2}{n} \mathcal{T}_{n+2}^{\prime}(y) y$. This gives:

$$
\mathcal{T}_{n+2}(y)+\frac{2}{n} \mathcal{T}_{n+2}^{\prime}(y) y=\frac{\sum_{i=1}^{\infty} \frac{\Gamma\left(\frac{n}{2}+1\right)}{i ! \Gamma\left(\frac{n}{2}+i+1\right)}\left[\left(\frac{\lambda \bar{y}}{2 \sigma^{2}}\right)^{i}-\left(1+\frac{2 i}{n}\right)\left(\frac{\lambda y}{2 \sigma^{2}}\right)^{i}\right]}{\sum_{i=0}^{\infty} \frac{\Gamma\left(\frac{n}{2}+1\right)}{i ! \Gamma\left(\frac{n}{2}+i+1\right)}\left(\frac{\lambda \bar{y}}{2 \sigma^{2}}\right)^{i}}
$$

See Appendix N for more details of the algebra.

For $f$ we use the characterization in equation (6) in term of modified Bessel functions of the first and second kind. These functions have a power series representation, which we use 
to solve for the two unknown constants $C_{1}, C_{2}$. This gives:

$$
\begin{aligned}
& f(y)=\left[\frac{\left(\frac{\lambda y}{2 \sigma^{2}}\right)^{\left(\frac{n}{2}-1\right)} \sum_{i=0}^{\infty} \beta_{i, \frac{n}{2}-1}\left(\frac{\lambda y}{2 \sigma^{2}}\right)^{i}}{\left(\frac{\lambda \bar{y}}{2 \sigma^{2}}\right)^{\left(\frac{n}{2}-1\right)} \sum_{i=0}^{\infty} \beta_{i, \frac{n}{2}-1}\left(\frac{\lambda \bar{y}}{2 \sigma^{2}}\right)^{i}}-\frac{\sum_{i=0}^{\infty} \beta_{i, 1-\frac{n}{2}}\left(\frac{\lambda y}{2 \sigma^{2}}\right)^{i}}{\sum_{i=0}^{\infty} \beta_{i, 1-\frac{n}{2}}\left(\frac{\lambda \bar{y}}{2 \sigma^{2}}\right)^{i}}\right] / \\
& {\left[\frac{\left(\frac{\lambda \bar{y}}{2 \sigma^{2}}\right)^{\left(\frac{n}{2}-1\right)} \sum_{i=0}^{\infty} \beta_{i, \frac{n}{2}-1} \frac{\bar{y}}{i+n / 2}\left(\frac{\lambda \bar{y}}{2 \sigma^{2}}\right)^{i}}{\left(\frac{\lambda \bar{y}}{2 \sigma^{2}}\right)^{\left(\frac{n}{2}-1\right)} \sum_{i=0}^{\infty} \beta_{i, \frac{n}{2}-1}\left(\frac{\lambda \bar{y}}{2 \sigma^{2}}\right)^{i}}-\frac{\sum_{i=0}^{\infty} \beta_{i, 1-\frac{n}{2}} \frac{\bar{y}}{i+1}\left(\frac{\lambda \bar{y}}{2 \sigma^{2}}\right)^{i}}{\sum_{i=0}^{\infty} \beta_{i, 1-\frac{n}{2}}\left(\frac{\lambda \bar{y}}{2 \sigma^{2}}\right)^{i}}\right]}
\end{aligned}
$$

where the two sequence of coefficients $\beta$ are defined in term of $\Gamma$ functions as

$$
\beta_{i, \frac{n}{2}-1} \equiv \frac{1}{i ! \Gamma(i+n / 2)} \text { and } \beta_{i, 1-\frac{n}{2}} \equiv \frac{1}{i ! \Gamma(i+2-n / 2)} \text { for } i=0,1,2, \ldots
$$

Due to a singularity on the power expansion of the modified Bessel function of the second kind, the expression in equation (27) holds for all real $n \geq 1$, except when $n$ is an even natural number. Yet the expression is continuous on $n$. See Appendix C for a step-by-step derivation.

Finally, using equation (26) and equation (27), rearranging terms and solving the resulting integrals we have:

Lemma 4 The equality equation (22) is equivalent to

$$
\begin{aligned}
& \sum_{j=1}^{\infty} \frac{\gamma_{j} \frac{1}{1+j}}{\sum_{s=0}^{\infty} \gamma_{s} \frac{1}{1+s}} j=\sum_{j=1}^{\infty} \gamma_{j}\left(1+\frac{2 j}{n}\right) \times \\
& \left(\left[\frac{\sum_{i=0}^{\infty} \xi_{i} \frac{1}{\frac{n}{2}+i+j}}{\sum_{i=0}^{\infty} \xi_{i}}-\frac{\sum_{i=0}^{\infty} \rho_{i} \frac{1}{i+1+j}}{\sum_{i=0}^{\infty} \rho_{i}}\right] /\left[\frac{\sum_{i=0}^{\infty} \xi_{i} \frac{1}{\frac{n}{2}+i}}{\sum_{i=0}^{\infty} \xi_{i}}-\frac{\sum_{i=0}^{\infty} \rho_{i} \frac{1}{i+1}}{\sum_{i=0}^{\infty} \rho_{i}}\right]\right),
\end{aligned}
$$

where the sequences $\left\{\gamma_{j}, \xi_{j}, \rho_{j}\right\}_{j=0}^{\infty}$ are defined as

$$
\begin{aligned}
\gamma_{j} & \equiv \frac{\Gamma\left(\frac{n}{2}+1\right)}{j ! \Gamma\left(\frac{n}{2}+1+j\right)}\left(\frac{\lambda \bar{y}}{2 \sigma^{2}}\right)^{j}, \xi_{j} \equiv \frac{1}{j ! \Gamma\left(j+\frac{n}{2}\right)}\left(\frac{\lambda \bar{y}}{2 \sigma^{2}}\right)^{\left(\frac{n}{2}+j-1\right)} \text { and } \\
\rho_{j} & \equiv \frac{1}{j ! \Gamma\left(j+2-\frac{n}{2}\right)}\left(\frac{\lambda \bar{y}}{2 \sigma^{2}}\right)^{j}
\end{aligned}
$$

For each $n>1$ and $\lambda \bar{y} / \sigma^{2}>0$ one can numerically evaluate the right and left hand side of equation (29) and check that they are equal. This verification is straightforward since both sides are simple functions of convergent power series, which are arbitrary well approximated by a finite sum (a simple Matlab code for the verification, called solveMp0.m, is available on our websites). As explained above, for $n=2 k$ and $k \in \mathbb{N}$ and $k \geq 1$, so that $n$ is even, this expression should be understood as the limit for $n \rightarrow 2 k$ (or, numerically, as the sum for values of $n$ close to $2 k$ ). 
Proof. (of Proposition 8). The idea is to show that for any $n, \ell$

$$
\operatorname{Kur}\left(\Delta p_{i} ; \mu\right)=\operatorname{Kur}\left(\Delta p_{i} ;-\mu\right), N\left(\Delta p_{i}\right)(\mu)=N\left(\Delta p_{i}\right)(-\mu), \mathcal{M}(\delta ; \mu)=-\mathcal{M}(-\delta ;-\mu)
$$

for all $(\mu, \delta)$ in a neighborhood of $(0,0)$. Note that differentiating the last expression with respect to $\delta$, and evaluating it at $\delta=0$ we obtain that $\mathcal{M}^{\prime}(0 ; \mu)=\mathcal{M}^{\prime}(0 ;-\mu)$. Hence we have that $\operatorname{Kur}\left(\Delta p_{i} ; \cdot\right), N\left(\Delta p_{i}\right)(\cdot)$ and $\mathcal{M}^{\prime}(0 ; \cdot)$ are symmetric functions of inflation around $\mu=0$. Hence, if they are differentiable, they must have zero derivative with respect to inflation at zero inflation. Instead the symmetry in equation (31) follows from the symmetry on the firm's problem with respect to positive and negative drift. To understand where this symmetry comes from, we proceed in two steps. First we analyze the symmetry of the decision problem for the firm of Section 3.2. Second, we consider the approximation to the GE problem for values $\mu \neq 0$. All the arguments follow, essentially, a guess and verify strategy of a simple nature but with a heavy notation. Appendix M provides more details on the proof.

Proof. (of Proposition 9.) The proof proceeds by verification analyzing the condition for which every single firm for which $\|p\|^{2}=y \leq \bar{y}$ before the shock will find that $\|p-\iota \delta\|^{2} \geq \bar{y}$, where $\iota$ is a vector of ones. See Appendix J for a detailed derivation. 